\title{
G s.textem \\ The ubiquitination landscape of the Influenza A Virus polymerase
}

\section{Franziska Günl}

https://orcid.org/0000-0002-4841-4363

\section{Tim Krischuns}

https://orcid.org/0000-0003-2693-4701

\section{Lea Henschel}

Institute of Virology Muenster, Westfaelische-Wilhelms University Muenster

\section{Marius Wahrenburg}

Clinic for tumor orthopedics and sarcoma surgery, University Hospital Essen

\section{Hannes Drexler}

Max Planck Institute for Molecular Biomedicine, Mass Spectrometry Unit, 48149 Muenster https://orcid.org/0000-0002-8285-0644

\section{Sebastian Leidel}

Department of Chemistry, Biochemistry and Pharmaceutical Sciences, University of Bern

\section{Vlad Cojocaru}

Hubrecht Institute https://orcid.org/0000-0003-0886-3401

\section{Guiscard Seebohm}

University Hospital Münster https://orcid.org/0000-0001-9303-5373

\section{Alexander Mellmann}

University Hospital Münster https://orcid.org/0000-0002-0649-5185

\section{Martin Schwemmle}

University Medical Center Freiburg https://orcid.org/0000-0002-2972-6855

\section{Stephan Ludwig}

Institute of Virology (IVM), Westfaelische Wilhelms Universitaet, Muenster, Nordrhein-Westfalen, 48149 https://orcid.org/0000-0003-4490-3052

\section{Linda Brunotte ( $\sim$ brunotte@uni-muenster.de)}

Institute of Virology Muenster, Westfaelische-Wilhelms University Muenster https://orcid.org/00000001-8074-7400

\section{Article}

\section{Keywords:}

Posted Date: February 2nd, 2022 
DOI: https://doi.org/10.21203/rs.3.rs-1283980/v1

License: (c) (1) This work is licensed under a Creative Commons Attribution 4.0 International License. Read Full License 
1 The ubiquitination landscape of the Influenza A Virus polymerase

2 Franziska Günl ${ }^{1}$, Tim Krischuns ${ }^{1,3}$, Lea Henschel ${ }^{1}$, Marius Wahrenburg ${ }^{1,4}$, Hannes C.A.

3 Drexler $^{5}$, Sebastian A. Leidel ${ }^{6}$, Vlad Cojocaru ${ }^{7,8}$, Guiscard Seebohm ${ }^{9}$, Alexander Mellmann ${ }^{2,10}$,

4 Martin Schwemmle ${ }^{11}$, Stephan Ludwig ${ }^{1,2}$, Linda Brunotte ${ }^{1,2}$

${ }^{1}$ Institute of Virology Muenster, Westfaelische-Wilhelms University Muenster, Münster,

8 Germany

$9 \quad{ }^{2}$ Interdisciplinary Center for Clinical Research (IZKF), Westfaelische-Wilhelms University 10 Muenster, Münster, Germany

$11{ }^{3}$ Current affiliation: RNA Biology and Influenza Virus Unit, Institute Pasteur, CNRS UMR3569, 12 Université de Paris, Paris, France

$13{ }^{4}$ Clinic for tumor orthopedics and sarcoma surgery, University Hospital Essen, Germany

14 BBioanalytical Mass Spectrometry, Max Planck Institute for Molecular Biomedicine, Muenster, 15 Germany

$16{ }^{6}$ Department of Chemistry, Biochemistry and Pharmaceutical Sciences, University of Bern, 17 Bern, Switzerland

$18{ }^{7}$ Department of Cell and Developmental Biology, Max Planck Institute for Molecular 19 Biomedicine, Muenster, Germany

208 In silico Biomolecular Structure and Dynamics Group, Hubrecht Institute, Utrecht, the 21 Netherlands

$22{ }^{9}$ Cellular Electrophysiology and Molecular Biology, Institute for Genetics of Heart Diseases 23 (IfGH), Department of Cardiovascular Medicine, Westfaelische-Wilhelms University Muenster, 24 Muenster, Germany

$25{ }^{10}$ Institute of Hygiene, University Hospital Muenster, Westfaelische-Wilhelms University 26 Muenster, Muenster, Germany

$27 \quad{ }^{11}$ Institute of Virology, University Medical Center Freiburg, Freiburg, Germany 28

*Corresponding Author: Dr. Linda Brunotte; brunotte@uni-muenster.de; +49 (0) 25183 $30 \quad 52213$

31 
During influenza A virus (IAV) infections, viral proteins are targeted by cellular E3 ligases for modification with ubiquitin. Here, we decipher and functionally explore the ubiquitination landscape of the IAV polymerase during infection of human alveolar epithelial cells by applying mass spectrometry analysis of immuno-purified K- $\varepsilon-G G$ (di-glycyl)-remnant-bearing peptides. We identified 59 modified lysines across all three subunits, PB2, PB1, PA, of the viral polymerase of which 17 distinctively affected mRNA transcription, vRNA replication and the generation of recombinant viruses via non-proteolytic mechanisms.

Moreover, further functional analysis revealed that ubiquitination at K578 in the PB1 thumb domain is mechanistically linked to the dynamic structural transitions of the viral polymerase that are required for vRNA replication. Mutations K578A and K578R impeded cRNA stabilization and vRNA synthesis, respectively, and affected NP binding as well as polymerase dimerization. Collectively, our results indicate that ubiquitin-mediated disruption of the chargedependent interaction between PB1-K578 and PB2-E72 is required to coordinate polymerase dimerization and facilitate VRNA replication, which demonstrates that IAV exploits the cellular ubiquitin system to modulate the activity of the viral polymerase for the regulation of viral replication.

\section{Introduction}

Influenza A viruses (IAV) are respiratory pathogens of the Orthomyxoviridae family that pose a substantial threat to global health through seasonal epidemics and recurring pandemics. The IAV genome consists of eight segments of negative-sense single-strand RNA (vRNA). Each vRNA is encapsidated by multiple copies of the nucleoprotein (NP) and one copy of the trimeric viral RNA-dependent RNA polymerase (RdRP), composed of the subunits PB2, PB1 and PA ${ }^{1,2}$ to form viral ribonucleoprotein (vRNPs) complexes ${ }^{3}$. Transcription of viral mRNAs and replication of the viral genome is mediated by the VRNP associated RdRPs and occurs in the cell nucleus. While mRNA synthesis is performed by the incoming vRNPs following capsnatching from host derived pre-mRNAs ${ }^{4-6}$, vRNA replication depends on newly produced viral proteins and proceeds in a primer-independent manner that involves the generation of full-length positive-sense complementary RNA (cRNA) intermediates, which serve as templates for vRNA synthesis ${ }^{7}$.

Structural investigations revealed that the IAV RdRP is a flexible and highly dynamic protein complex that adopts different conformations during mRNA transcription and vRNA replication ${ }^{5,8}$. Moreover, the current model for vRNA replication suggests that the transition from the transcriptase to the replicase is promoted by the interaction with a second viral polymerase forming an asymmetric dimer ${ }^{9,10}$. In addition, the formation of the asymmetric dimer facilitates encapsidation of the nascent cRNA strand. In the second step, initiation of the vRNA synthesis from the cRNA template requires formation of a symmetric polymerase dimer 
dimerization is required to maintain the optimal balance between mRNA transcription and vRNA replication during the viral life cycle and is suggested to be facilitated by viral as well as cellular factors. In particular, host-mediated post-translational modifications (PTM) of viral proteins were proposed as dynamic molecular switches to fine-tune the activity of the RdRP ${ }^{13,14}$. Indeed, phosphorylation ${ }^{15}$, ADP-ribosylation or acetylation ${ }^{16-18}$ as well as linkage of ubiquitin-like modifiers (UBL), such as SUMO 1-3, NEDD8, ISG15 and ubiquitin (UB), to the polymerase subunits and NP have been reported. Among UBLs, ubiquitination is the most abundant modification in human cells and regulates the functionality and longevity of a wide range of proteins and has been described for all subunits of the IAV RdRP with both pro- and antiviral outcomes ${ }^{19-22}$. However, the biological impact of site-specific modifications for the processes of viral mRNA transcription and genome replication has remained largely unknown. In this study, we performed mass spectrometry (MS) analyses of immuno-selected, K- $\varepsilon$-GG(di-glycyl)-enriched viral proteins purified from infected human lung epithelial cells to unravel the ubiquitination landscape of the IAV polymerase. We identified 59 modified lysines across all subunits of the IAV RdRP, with UB being the most abundant UBL. Mutational analysis and generation of recombinant viruses by reverse genetics revealed that ubiquitination at position PB1-K578 in the PB1-thumb domain regulates the spatio-temporal positioning of an unstructured N-terminal loop in the PB2 subunit by charge-dependent interaction with PB2E72 and thereby participates in the coordination of polymerase dimerization, NP binding and the two steps of vRNA replication.

\section{Results}

\section{Unraveling the ubiquitination landscape of the IAV polymerase during infection}

Western blot analysis of co-precipitated strep-tagged RdRP subunits, PB2, PB1 and PA, with co-expressed UBLs revealed abundant UB modifications on all three subunits, which is in line with previous reports (Fig. 1A and Suppl. Fig. 1) ${ }^{21}$. In contrast, NEDD8 was only detected on PA and ISG15 was not detected at all (Fig. 1B and C).

To determine the specific location of the UBL modified lysines within the IAV polymerase during the early phase of the viral infection cycle in human alveolar epithelial cells, we infected A549 cells with the human IAV strain A/1933/WSN(H1N1) (WSN) at a multiplicity of infection (MOI) of 20 for $5 \mathrm{~h}$, followed by immuno-selection of trypsin digested peptides harboring di-glycyl remnants and MS analysis (Fig. 1D and E).

In total, we identified 59 lysines with di-glycyl remnants distributed across all three subunits of the polymerase, of which 22 were located in the PA and PB2 subunits, respectively, and 15 in PB1 (Table 1). The majority of the modified lysines were highly conserved among IAV strains from birds, swine and humans (Fig. 1F-H). To assess surface accessibility and spatial distribution of the modified lysines within the described conformational states, we generated sequence-adapted three-dimensional structure models of the trimeric WSN RdRP bound to promoters of vRNA (Fig. 2A) or cRNA (Fig. 2B) by homology modeling $8,9,11,23$. The majority of 
the modified lysines were exposed on the surface of the polymerase trimer in at least one of the conformations, suggesting that modification by E3 ligases can occur in the context of the individual proteins as well as the active polymerase trimer (summarized in table 1). However, some lysines in PB1 were located within the inaccessible hydrophobic core, suggesting that modification occurred prior to the assembly of the polymerase trimer. Importantly, many lysines were located in functionally described subdomains of the polymerase (Fig. 2C). In the PB2 subunit, the modified lysines were located in two main clusters: the N-terminal domain, which extends to the N2 domain, and the combined C-terminal 627-NLD domain. Both regions are crucial for interactions with NP, PA and PB1 ${ }^{24-31}$. Moreover, several modified lysines were located in regions implicated in polymerase dimerization ${ }^{9,11}$. Only three modified lysines were detected in the flexible and exposed mid, cap-binding (CapB), and linker domains. Several modified lysines were embedded in nuclear localization signals (NLS) or mediated interaction with the cellular nuclear import protein importin- $\alpha$, suggesting that UBLs affect nuclearcytoplasmic shuttling of PB2 proteins or the RdRP complex ${ }^{32,33}$.

In PA, seven di-glycyl-carrying lysines resided in the N-terminal endonuclease domain (Fig. 2C, middle panel), including the catalytic residue $\mathrm{K} 134^{34-36}$, Interestingly, four lysines directly faced towards the PB2-CapB domain in the transcriptase conformation of the trimeric polymerase, including K102 and K104, which reportedly interact with the 5'-capped mRNAprimer during transcription ${ }^{36}$, suggesting that the cap snatching process is a putative target for regulation by ubiquitination. Three modified residues were located in the PA linker region. Within the PA C-terminus three modified lysines were found in the region involved in the formation of the symmetric dimer ${ }^{9,11,12}$ and six within the interaction site to cellular RNA polymerase II (pol-II) ${ }^{28,37}$. In both, PA and PB1, several modified lysines were located in close proximity to the binding pocket of the 5'hook of the vRNA and cRNA templates ${ }^{28,38-41}$. The central polymerase subunit PB1 exhibited the lowest number of modified lysines in our analysis. Further, only 7 out of 15 lysines were surface exposed, while the rest was located in the hydrophobic core or covered by the other subunits within the trimeric complex (Fig. $2 \mathrm{~A}$ and $\mathrm{B}$, lower panel). Residue $\mathrm{K} 480$ is located at the rim of the NTP tunnel, where it has been shown to participate in NTP binding during RNA synthesis ${ }^{28,41}$. K578 resides in the PB1 thumb domain, which is part of the vRNA template exit tunnel during mRNA transcription. In addition, it directly points towards the PB2-N1-domain and is located in close proximity to the interface of the symmetric dimer as well as the secondary vRNA $3^{\prime}$-end binding site ${ }^{5,11,42}$. In summary, our results provide the first comprehensive map of UB modifications that are acquired across all subunits of the IAV viral polymerase during the first $5 \mathrm{~h}$ p.i. of human lung epithelial cells.

\section{Substitution of modified lysines impacts polymerase functions}

To assess the functional impacts of the site-specific modifications identified in our analysis, we substituted each lysine with a non-charged alanine $(A)$ or a positively charged arginine $(R)$, which both lack the UB acceptor function, and determined polymerase activity (Fig. 3A), 
intracellular localization and generation of infectious recombinant viruses (Fig. 3B). Overall, introduction of $A$ was tolerated at 23 out of 56 positions without affecting the polymerase activity (Suppl. Fig. 2A-C). At 16 sites, A mutations significantly changed the polymerase

154 activity, however, this effect was reverted by the $\mathrm{R}$ mutation, which suggests that it was the positive charge of the lysine rather than the presence of the UB that determined its role for polymerase activity (Suppl. Fig. 2D-F). For the 17 remaining positions, A and $R$ substitution significantly up- or down-regulated the polymerase activity, which corroborated their function as UB acceptor sites and suggested a regulatory role of the modification on the activity of the viral polymerase. This was further supported by the location of these lysines within binding sites to NP, the other polymerase subunits and pol-II as well as in the NLS and RNA binding motifs, which are essential domains that are crucial for polymerase activity (Fig. 3C-E). Notably, previously reported NEDD8 and UB acceptor sites, such as K699 ${ }^{43}$ K752 and K482 22 in PB2, were also among the identified regulatory lysines. We identified two positions that indicated an antiviral effect of the UB modifications. This included PB2-K157 in the mRNA exit tunnel and PB1-K635 in the thumb domain, which demonstrated increased polymerase activity upon $A$ and $R$ mutation and also resulted in the generation of stable recombinant viruses (Figure $3 \mathrm{C}$ and $\mathrm{E}$ ). Interestingly, mutations at eight positions distributed on all subunits abrogated or reduced polymerase activity but still gave rise to recombinant viruses (Figure 3CE bottom panels, table 1). We speculated that this discrepancy was facilitated by residual vRNA replication. To confirm this hypothesis, we determined synthesis of the different viral RNA species with primers specific for mRNA and cRNA using qRT-PCR. Indeed, all mutations ablated mRNA transcription but retained at least $40 \%$ cRNA synthesis activity (Fig. 3F and $\mathrm{H}$ ). In the case of PB1-K360A cRNA synthesis was even upregulated to more than $250 \%$ compared to the wild type polymerase (Fig. $3 \mathrm{H}$ ). This indicate that UB modifications at these positions are specifically required for mRNA transcription. Residue PA-K536, which resides within a putative RNA template binding groove ${ }^{39,40}$ demonstrated immediate reversion to $\mathrm{K} 536$ during virus rescue. This suggests that neither a constantly neutral nor a positively charged residue is tolerated at this position and indicates a requirement for a lysine as a UB acceptor site to achieve neutralization of the positive charge at a distinct time point during viral replication.

Positions in domains with catalytic activity, such as the endonuclease in PA (K134) and the polymerase catalytic cavity in PB1 (K229, K347, K471) were in general highly sensitive to substitution with $\mathrm{A}$ or $\mathrm{R}$ leading to loss of polymerase activity and lack of recombinant virus generation despite robust protein expression (Fig. 3D and E, Suppl. Fig. 2H and I). PB2-K617, located in a putative NP binding region ${ }^{27,44}$, was likewise sensitive to mutation, pointing towards a pro-viral function of the UB modification.

The mutations PB2-K699R and PA-K615A did not support the generation of recombinant virus despite increased polymerase activity upon loss of the respective lysine, suggesting a curtailing role of the modification for RNA synthesis as well as a supportive function for later 
steps in the viral life cycle, which might be hindered by the mutations. In the case of PB1-K578, $A$ and $R$ mutations strongly elevated the polymerase activity (Fig. 3E). However, while K578A gave rise to stable recombinant virus in all replicates, rescue with $\mathrm{K} 578 \mathrm{R}$ resulted in infectious plaques in only one out of three replicates, indicating distinct functional outcomes of the mutations. Some mutations also affected the intracellular localization of the polymerase subunits. We observed an increased cytoplasmatic localization of PB2-K482R and PB2-K699R (Fig. 3I), which are both known to be involved in nuclear-cytoplasmic shuttling of PB2 ${ }^{32,45}$. In addition, PA-K635A resulted in an increased translocation of PA into the nucleus (Fig. 3J and K). In summary, our functional analysis reveals that host-derived site-specific UB modifications in the polymerase subunits confer positive and negative effects on the activities of the viral polymerase. Furthermore, our results provide evidence that several modifications distinctively impact viral mRNA transcription or vRNA replication.

Mutation of PB1-K578 impairs NP binding, polymerase dimerization and vRNA replication

In contrast to K578A, only one out of three independent rescue attempts resulted in the generation of infectious virus (R1) with the PB1-K578R mutation, despite the increased polymerase activity (Fig. 3E). The low stability of K578R was further corroborated by reduced hemagglutination inhibition $(\mathrm{HI})$ titers and low viral titers reaching less than $10^{4} \mathrm{PFU} / \mathrm{ml}$ after virus rescue (Fig. 4A and Suppl. Fig. 3A, left). However, virus titers increased to comparable levels of the wild type virus after one additional passage of the rescue supernatant (Fig. 4B), indicating either reversion of the mutation or introduction of compensatory mutations. Interestingly, despite the lack of infectious virus, purification of viral RNA from the rescue supernatants indicated the generation of non-infectious particles in R2 and R3. Deep sequencing revealed a high prevalence of adenines at nucleotide positions 1732-1734 in R1 demonstrating a high frequency of reversion from 578R to K (Fig. 4D). In contrast, in the noninfectious replicates $\mathrm{R} 2$ and $\mathrm{R} 3$ the distribution of nucleotides at these positions was more heterogeneous, with $0 \%$ and $1 \%$ adenines at the first position in $\mathrm{R} 2$ and $\mathrm{R} 3$, respectively, suggesting absence or very low abundance of $578 \mathrm{~K}$. Of note, R2 and R3 contained fractions encoding for $578 \mathrm{~A}$ or $\mathrm{Q}$, but nevertheless failed to produce infectious virus even after additional passaging. Compensatory mutations in the polymerase or other genes were not observed. After one passage, R1 completely reverted to $\mathrm{K} 578$ and remained stable for 4 additional passages on A549 cells, which was also confirmed by wild type-like viral and $\mathrm{HI}$ titers (Fig. 4B and Suppl. Fig. 3A, right panel). These results indicate strong positive selection of PB1-K578 and suggest that mutation of this residue to $R$ interferes with some step of viral replication. In contrast, PB1-K578A exhibited neither reduced viral and HI titers (Fig. 4C, Suppl. Fig. 3A) nor sequence instability for up to 5 passages after the rescue. Determining the abundance of the individual vRNA segments within virus particles from the rescue supernatants of PB1-K578R, PB1-K578A and the wild type virus demonstrated that all eight vRNA segments were present 
in viral particles from the rescues giving rise to viable virus (Fig. 4E). In contrast, the noninfectious viral particles from R2 and R3 of the PB1-K578R rescue contained incomplete viral genomes, implying that defective vRNA replication accounts for the generation of noninfectious virus particles in these replicates. To investigate the dynamics of vRNA replication and virus amplification during rescue of the wild type virus and the PB1-K578R mutant, we determined the amounts of segment-specific vRNAs in the transfected cells at $16 \mathrm{~h}$ and $48 \mathrm{~h}$ post transfection (h p.t.) of the 8 rescue plasmids. Interestingly, at $16 \mathrm{~h} \mathrm{p.t.} \mathrm{the} \mathrm{levels} \mathrm{of} 7 \mathrm{vRNA}$ segments (excluding HA) were significantly higher for the K578R mutant compared to the wild type (Fig. 4F). However, at $48 \mathrm{~h}$ p.t. this advantage was lost and vRNA levels of all segments were strongly reduced compared to the wild type (Fig. 4G), confirming that PB1-K578R causes a pronounced defect in the amplification of vRNA. Interestingly, the strongest reduction could be seen for segments PB1 and PA, which resemble the missing genome segments in the noninfectious particles of R2 and R3 (Fig. 4E). To discriminate which step of vRNA replication was affected by K578R, we next compared the ability of reconstituted wild type and mutant vRNPs to stabilize and transcribe a cRNA template that was provided by viral infection (Fig. $4 \mathrm{H}$ ). Cells were first transfected with plasmids expressing the PB2, PB1, PA and NP for $24 \mathrm{~h}$ followed by infection with wild type virus in presence of cycloheximide to inhibit translation of the viral proteins. Measuring the levels of cRNA demonstrated that K578A and K578R both resulted in a significant reduction of cRNA levels, albeit more pronounced with the $\mathrm{R}$ mutant, suggesting a defect in cRNP stabilization (Fig. 4I). Analysis of vRNA levels further revealed that the K578A-mutant synthesized significantly less amounts of vRNA, suggesting a defect in cRNA to vRNA transcription (Fig. 4J). These results provide evidence, that PB1-K578 is crucial for cRNA stabilization and vRNA synthesis from the cRNA template and indicate that non-modified and modified K578 are required for distinct steps in the process of vRNA replication. Accumulating evidence suggests that vRNA replication relies on the presence of NP and

involves polymerase dimerization ${ }^{9,11,46-49}$. Based on the previous results, we speculated that mutation of PB1-K578 modulates binding of NP to the polymerase or RdRP dimerization and thereby causes the defects in cRNA stabilization and vRNA synthesis. Co-immunoprecipitation of strep-tagged NP with the viral polymerase demonstrated that PB1-K578A, but not PB1K578R, destabilized the interaction of NP with the trimeric polymerase (Fig. 4K and Suppl. Fig. S3B). In addition, dimerization between two heterologous polymerase trimers was reduced with PB1-K578A and the previously reported dimerization deficient PB2 triple mutant PB2-7173A (Fig. 4L) ${ }^{11,12}$. These results provide evidence that PB1-K578 participates in the interaction between NP and the viral polymerase as well as polymerase dimerization during vRNA replication.

\section{PB1-K578 interacts with PB2-E72 in a charge-dependent manner}

266 The selection pressure for PB1-K578 (Fig. 4D) suggested a requirement for a dynamic UB 267 modification at this residue. Digestion of wild type and K578A-mutated PB1 with the UB- 
specific peptidase USP2 ${ }^{50}$ demonstrated that K578A was less sensitive to de-ubiquitination compared to the wild type protein, providing additional evidence for modification of K578 by UB and not NEDD8 (Fig. 5A). To obtain structural insights on how UB modification of PB1K578 could affect vRNA replication through NP binding and RdRP dimerization, we analyzed the structural environment of this residue in more detail. PB1-K578 is part of a surface exposed $\alpha$-helix within the PB1 thumb domain and is directly oriented towards the negatively charged residue PB2-E72, which resides at the tip of a mostly unstructured and flexible loop in the PB2$\mathrm{N} 1$ domain (AA 63-82) (Fig. 5B and C) ${ }^{11,12}$. The PB2 N-terminus was reported to participate in NP binding ${ }^{44}$ (Fig. 2C) and the PB2 loop harbors several residues that mediate the formation of the symmetric polymerase dimer. Specifically, neighboring residues PB2-R70, Q73 and T76 were shown to establish direct interactions to positions Q374 and D347 in the PA subunit of a second, putatively trans-activating, polymerase (Fig. 5C) ${ }^{11}$. The amino acid sequences of the PB1-helix and the PB2 loop are both highly conserved among IAV strains including common laboratory strains as well as natural isolates of IAV strains of human, swine and avian origin (Suppl. Fig. 4A and B). At position 578, lysine is the predominant amino acid in PB1 sequences derived from human (98.82\%), avian (99.26\%) and swine (75.18\%) isolates (Fig. 5D). Only $0.03 \%$ of avian IAV strains contained A578, while it was not present in human and swine IAV isolates, suggesting low evolutionary stability in nature. Interestingly, of the analyzed swine PB1 sequences $12.47 \%$ encoded for R578 and $12 \%$ for S578, a putative acceptor site for phosphorylation. To assess the functional conservation of PB1-K578 we substituted PB1-K578 with $A$ and $R$ in PB1 of IAV strains PR8 (H1N1) and SC35M (H7N7), which resulted in enhanced polymerase activity and resembled the results of the WSN RdRP (Fig. 5E). Furthermore, we identified structural resemblance of the PB1-PB2 interface in the polymerases of influenza B and C as well as bat influenza viruses (Suppl. Fig. 4C-E). Finally, we explored the charge dependency of the putative interaction between PB1-K578 and PB2-E72 by insertion of different charged and non-charged residues at both positions and assessed the impact on polymerase activity and recombinant virus generation. This revealed, that noncharged or negatively charged residues at PB1-578 resulted in increased polymerase activity and recombinant viruses. In contrast, for PB2-E72 neutral A and positively charged $R$ increased polymerase activity, confirming the charge-dependent nature of the interaction between PB1-K578 and PB2-E72 (Suppl. Fig. 4F and G). Of note, neutralization of the positively charged residue PB2-R101 conferred similar effects, suggesting that PB1-K578 and PB2-R101 cooperatively stabilize the PB2 loop (Suppl. Fig. 4G and H).

PB1-K578 controls the spatio-temporal position of the PB2 loop in a charge-dependent manner

304 To overcome the static nature of our mutational analysis for the determination of possible structural impacts mediated by the disruption of the PB1-K578 interaction to PB2-E72, we performed dynamic secondary structure analyses and local molecular dynamic simulation with 
the WSN-adapted 3D model of the cRNA promoter-bound IAV polymerase. The results demonstrated that the charge at position PB1-578 majorly determines the spatial structure of the PB2 loop through changing the helical and $\beta$-sheet content in regions a (AA 63-67) and b (AA 78-82) (Fig. 6A and Suppl Fig. 4I). Moreover, the results of the molecular simulation confirmed the charge-dependent interaction between the positively charged PB1-K578 and K578R with the negatively charged PB2-E72, which was absent with PB1-K578A (Fig. 6B). This analysis further indicated that $\mathrm{K} 578 \mathrm{R}$ resulted in prolonged and less dynamic interaction with residues PB2-Q73 and R101 compared to K578 (Fig. 6C and D), which likely leads to a stabilization of the PB2 loop and thereby promotes the interaction to the trans-activating polymerase that is required for the formation of the symmetric dimer. These results suggest that the defect in vRNA replication of the K578R mutant is caused by restricted mobility of the PB2 loop and thereby constitutes the selection pressure for reversion to K578. Presumably, neutralization of $\mathrm{K} 578$ by ubiquitination provides a molecular switch that determines the stability of the PB2 loop, which facilitates transition of the transcriptase to the replicase and regulates both steps of vRNA replication (Fig 6E).

\section{Discussion}

Here, we unraveled the first comprehensive landscape of site-specific ubiquitin modification in the IAV viral polymerase during infection. The combination of di-glycyl-targeted peptide enrichment and MS analysis resulted in the identification of $59 \mathrm{UBL}$ modified lysines across all three subunits of the polymerase, the majority of which have not been described nor functionally characterized before. Most lysines were highly conserved in IAV isolates from humans, birds and pigs and resided within functionally and structurally important regions, emphasizing their putative impact on viral replication. Although di-glycyl remnants derive from different UBL modifiers, we demonstrated that ubiquitin is the most abundantly detected modification in the viral polymerase, which is consistent with earlier reports ${ }^{21,43}$. Several other groups reported site-specific UBL modifications in the IAV polymerase and NP with effects on viral replication. However, these effects were commonly attributed to proteasomal degradation or destabilization of the modified viral proteins, while specific effects on the polymerase functions were not reported ${ }^{20,43,51}$. Our work provides a new perspective on UB modifications of the IAV polymerase and demonstrates that such modifications confer site-specific effects on the activity of the viral polymerase and the generation of recombinant viruses through nonproteolytic mechanisms, which substantiates that IAV exploits the cellular UB system to optimize viral replication. We describe no less than 17 UB acceptor sites in the viral polymerase, which positively or negatively affect the activity of the viral polymerase on the level of intracellular localization, mRNA transcription, vRNA replication and generation of recombinant viruses upon substitution with $A$ and $R$. Interestingly, we found that several mutations resulted in the abrogation of mRNA transcription, however, with minor effects on cRNA synthesis. This provides strong evidence for a broad spectrum of functional roles of site- 
specific ubiquitination in the temporal coordination of both processes and emphasizes that the regulatory impact of host-derived and site-specific UB modifications on the viral polymerase activity exceeds our current understanding and thus requires further investigation. In addition to the description of new ubiquitination sites within the IAV polymerase and their functional characterization, we provide compelling evidence that the UB acceptor site PB1K578 plays a crucial role for mRNA transcription and vRNA replication. The importance of K578 was revealed by mutational substitution with $A$ and $R$, both of which affected the synthesis of viral mRNA and vRNA synthesis as well as viral replication, yet with very different outcomes. According to the current model for mRNA transcription ${ }^{5}$, we speculate that the increased polymerase activity of the PB1-K578A/R mutants observed in the reconstitution assay (Fig. 3E) derives from an altered repositioning of the PB1 thumb domain and the PB2$\mathrm{N} 1$ domain, which alleviates blockage of the template exit channel by PB2 (AA 80-90) in the pre-initiation complex of the transcriptase and thereby promotes mRNA elongation. While PB1-K578A supported the generation of a stable recombinant virus, we were unable to rescue a PB1-K578R virus. Instead, we observed a strong positive selection pressure for reversion, which provided evidence that a constant positive charge at this position confers a detrimental defect for viral replication. This result emphasizes the necessity for ubiquitination of this residue during viral replication. We found that $\mathrm{K} 578 \mathrm{R}$ caused a defect in the replication of vRNA at the step of nascent cRNA encapsidation and resulted in the loss of vRNA amplification during viral rescue as well as incomplete viral genomes in non-infectious virus particles (Fig. 4). Interestingly, also K578A inherits a defect in vRNA replication at the step of cRNA to vRNA synthesis, which suggests that vRNA replication is equally dependent on nonmodified and ubiquitinated $\mathrm{K} 578$. Our data further revealed that $\mathrm{K} 578 \mathrm{~A}$ resulted in reduced binding of the polymerase trimer to NP and impeded polymerase dimerization, suggesting that ubiquitination of $\mathrm{K} 578$ coordinates these steps in a timely manner and thereby also regulates vRNA replication. Finally, dynamic molecular structure modeling provided insights into the underlying structural mechanism and revealed that PB1-K578 determines the spatio-temporal position of an unstructured loop in the PB2 N-terminus. Importantly, this loop harbors several residues that are directly involved in the interaction to the second polymerase in the symmetric dimer that is required for the initiation step of vRNA replication ${ }^{11}$. PB1-K578 interacts with residue PB2-E72, which resides at the tip of this loop, in a charge dependent manner. This suggests that ubiquitination is exploited to neutralize the positive charge of K578, which is corroborated by the decreased dimerization of the PB1-K578A mutant. Since the asymmetric dimer uses a different interaction interface, we speculate that ubiquitination of PB1-K578 disturbs the formation of the symmetric dimer but not the asymmetric dimer. Taken together, we propose that ubiquitination of K578 is required to prevent NP binding to newly synthesized trimeric polymerases and to achieve correct positioning of the PB2 loop that prevents the premature assembly of the symmetric dimer. This would deprive the pool of free polymerases and disfavor the formation of the asymmetric dimer leading to defective vRNA replication, as 
demonstrated by the PB1-K578R mutant. In contrast, we assume that the trans-activating polymerase derives from the pool of non-modified free polymerases and supports dimerization via the symmetric interface, to facilitate template realignment and vRNA transcription from the cRNA template (Fig. 6E).

389 Previous work demonstrated that among the more than 600 E3 Ubiquitin ligases UBR5, 390 STUB1/CHIP, HUWE1 and DDB1 interact with the viral replication machinery in infected cells ${ }^{14}$. However, siRNA mediated depletion did not provide convincing evidence for a role in PB1-K578 ubiquitination. Several groups have previously used comparative interactomics of the human ubiquitin proteasome system with viral proteins, genome-wide RNAi library screens or mass spectrometry-based interaction screens ${ }^{51-53}$ to identify candidate E3 ligases that facilitate ubiquitination of viral proteins. The results of our study can now be combined with such technologies to link cellular E3 ligases to site specific modifications in the viral polymerase and uncover new therapeutic targets. In conclusion, in addition to generating the comprehensive ubiquitin landscape of the viral polymerase, our results provide a novel and unexpected role of host-derived ubiquitination of residue PB1-K578 in the PB1 thumb domain for the coordination of NP binding, polymerase dimerization and VRNA replication. These novel findings advance our understanding of the enigmatic binding site of NP to the polymerase trimer and unravel the important role of the flexible N-terminal PB2 loop for the formation of the symmetric polymerase dimer.

404

\section{Acknowledgement}

406 We thank Ted Dawson, Edward Yeh and Gerrit Praefcke for providing plasmids. We owe our 407 thanks to Max Trauernicht, Lars Schmitz, Julius Kindsgrab, Josephine Friedrich, Hannah 408 Junginger, Nadja Rotte, Ramona Wördemann and Josua Janowski for support and Sebastian 409 Giese and Kevin Ciminski for helpful comments and discussions. We are grateful for support 410 by Geoffrey Chase for editing of the manuscript.

411 This work was funded by the German Research Foundation (DFG) (Grant BR5189/1-1 to LB; SCHW 632/21-1 to MS) and the Clinical Research Unit CRU342 (Project P6 to LB and SL; project P4 to AM), Further support was received by the Interdisciplinary Centre for Clinical 414 Research (IZKF) (Grant Bru2/015/19 to LB) of the Medical Faculty of the Westfaelische415 Wilhelms University and the Swiss National Science Foundation (SNF) (Grant 310030_184947 416 to $S A L)$.

\section{Author contributions}

419 LB, FG and TK designed and conceptualized the study. FG, TK, LH, MW performed experiments and functional assays including plasmid cloning, polymerase reconstitution assays, immune fluorescence analysis, viral rescues and protein interaction studies. TK, SL and HD were responsible for protein purification from infected cells and MS analysis. VC and 
further structural analysis. In silico dynamic structure modeling of the mutated viral polymerase and viral genome sequencing was performed by GS and AM, respectively. MS and SL provided laboratory infrastructure, plasmids and cell lines and supported writing and editing of the manuscript. LB and FG wrote the first draft of the manuscript and designed the figures with support from all co-authors.

\section{Declaration of interests}

The authors declare no competing interests.

\section{Figure titles and legends:}

Figure 1: Identification of site-specific UB modifications in the IAV polymerase

A-C: Western blot analysis of ubiquitin, NEDD8 and ISG15 modification of the IAV polymerase subunits. A549 (ubiquitin) or HEK293-T (NEDD8, ISG15) were transfected with expression plasmids for strep-tagged polymerase subunits (PB2, PB1 or PA) respectively, together with plasmids for the expression of UBLs bearing an HA- or His-tag. UBL modified polymerase subunits were precipitated under denaturing conditions using Strep-Tactin ${ }^{\circledR}$ bound sepharose beads. UBL modification was detected by western blot using anti-HA, anti-NEDD8 or anti-His antibodies as well as subunit-specific antibodies. D: Experimental outline for the identification of di-glycylated lysines using a di-glycyl-specific immunoselection coupled to mass spectrometry analysis. E: Sequence alignment of ubiquitin-like modifiers. The di-glycyl motif is highlighted in yellow together with the N-terminal amino acid, which determines trypsin cleavage (black box). F-H: Conservation analysis of the identified UBL acceptor lysines in PB2, PB1 and PA. PB2-K113, -K627; PB1-K278, -K586 are not depicted. PB2, PB1 and PA sequences from human, swine and avian IAV isolates were downloaded from NCBI (PB1 and PB2: 09/15/2017; PA: 10/01/2017) and analyzed for sequence identity. Relative frequency of lysines at the respective position in PB2 $(F), P B 1(G)$ and PA $(H)$ is depicted. In case of a lysine frequency below $75 \%$, the most abundant $A A$ is shown ( $R=$ Arginine).

\section{Figure 2: UB modified lysines reside in functional domains of the IAV polymerase}

A-B: 3D structural models of a WSN-adapted heterotrimeric IAV polymerase bound to vRNA (adapted from PDB: 4WSB) (A) or cRNA (adapted from PDB: 5EPI) (B) created by comparative homology modeling. Positions of lysines with diglycyl remnants are depicted in violet (PB2, yellow, upper panel), green (PA, violet, middle panel) and magenta (PB1, turquoise, lower panel). C: Linear models of PB2 (upper panel), PA (middle panel) and PB1 (lower panel) depicting the location of the identified di-glycylated lysines (K- $\square-G G$ position) in previously described functional domains. Bold numbers represent sites that are surface exposed in the heterotrimeric polymerase regardless of the bound RNA subtype. Underlined sites are part of domains involved in major repacking processes depending on the bound RNA. Numbers in light gray represent modified lysines that were not included in further functional analysis. 
Boundaries of functional domains (grey boxes) are depicted in the upper lane (\#AA).

464

465

466

467

468

469

470

471

472

473

474

475

476

477

478

479

480

481

482

483

484

485

486

487

488

489

490

491

492

493

494

495

496

497

498

499

500

501

Interaction sites to viral proteins PA (violet), PB1 (light green), PB2 (orange), NP (dark orange) and NEP (brown) and the cellular proteins pol-Il (dark green), importin (bright orange) and ANP32A (pink) are included. RNA interaction sites are depicted as follows: mRNA (grey) vRNA (white boxes) or cRNA (black boxes) and the 5'Cap-structures of cellular mRNAs (bright green boxes). Other functional motives include the catalytic domains of the polymerase in PB1 (light pink), NLS (violet) and the priming loop (dark green).

Figure 3: Mutational screen of modified lysines reveals distinct effects on mRNA transcription and vRNA replication

A: Schematic overview of the polymerase reconstitution assay. HEK293-T cells are cotransfected with expression plasmids for PB2, PB1, PA and NP together with two reporter plasmids for a firefly-encoding vRNA minigenome under control of the pol-I promoter and the Renilla luciferase mRNA under control of the pol-II promoter. FF activity correlates with the transcriptional activity of the reconstituted viral polymerase. Renilla activity serves as an internal transfection control. B: Overview of the reverse genetic system for the generation of recombinant influenza viruses. Co-cultures of HEK293-T and MDCK-II cells are co-transfected with the bi-directional pHW2000 plasmids encoding for the 8 viral genome segments. Viral mRNA and vRNA is generated from cellular pol-II and pol-I promoters, respectively. Rescued viruses are plaque purified, propagated on MDCK-II cells, and sequenced using Sanger sequencing. C-E: Polymerase reconstitution assay of the polymerase complex with $A / R$ substitutions of the UBL modified lysines in WSN-derived PB2 (C), PA (D) and PB1 (E). Relative FF activities are presented as the mean percentage activity using the wild type polymerase in three independent replicates $( \pm S E M)$. Statistical significance was assessed using a multiple comparison one-way ANOVA test ${ }^{* *} p \leq 0.01 ;{ }^{* * *} p \leq 0.001$; ${ }^{* * *} p \leq 0.0001$ ). Success (+) or failure (-) to generate recombinant viruses is depicted below. Virus rescue was considered negative if three independent rescue attempts failed. F-H: Quantification of FF mRNA and cRNA levels from the polymerase reconstitution assay using plasmids with the indicated mutations in PB2 (F), PA (G) or PB1 (H) using qRT-PCR. mRNA and cRNA levels of three independent replicates were detected using specific primers and are depicted relative to the wild type viral polymerase. Cellular GAPDH was used as housekeeping control. I-K: Effects of selected mutations on the subcellular localization of the respective polymerase proteins were investigated by immunofluorescence. A549 cells were transfected with plasmids expressing wild type or mutated versions of PB2 (I), PA individually (J) or in combination with HA-tagged PB1 (K). $24 \mathrm{~h}$ post transfection cells were fixed and analyzed using the indicated antibodies. Cell nuclei were visualize using DAPI.

Figure 4: Mutation of PB1-K578 affects cRNP stabilization and vRNA transcription by modulating NP binding and polymerase dimerization 
A-C: Viral titers of wild type and PB1-K578A/R viruses after rescue $(A)$ or passaging $(B-C)$, $\mathrm{n}=3$. Passaging was performed using $\mathrm{A} 549$ cells (MOI: 0.01 or 0.001 for the first passage in B) and viral titers were determined $48 \mathrm{~h}$ p.i. as plaque forming units (PFU) per ml. D - E: Detection of packaged vRNA segments isolated from released virus particles in the supernatants of the rescue experiments at $48 \mathrm{~h}$ p.t. vRNA segments were amplified with segment-specific primers, separated on $2 \%$ agarose gel $(E)$, and analyzed using deep sequencing. Percentage of nucleotides encoded as position 1732-34 encoding for K578 (D). F-G: Accumulation of vRNA segments in HEK293-T cells co-transfected with the pHW2000 plasmids at the indicated time points. vRNA levels were quantified using segment specific primers for RT-PCR and presented as the mean $n$-fold of WT ( \pm SEM), $n=3$. Cellular GAPDH was used as housekeeping control. Statistical significance was assessed using a multiple comparison one-way ANOVA test $\left({ }^{* *} p \leq 0.01 ;{ }^{* * *} p \leq 0.001 ;{ }^{* * * *} p \leq 0.0001\right)$. H: Schematic overview of the cRNP-stabilization assay. HEK293-T cells were transfected with plasmids expressing PB2, PB1, PA and NP. At $24 \mathrm{~h}$ p.t., cells were infected with wild type WSN virus (MOI: 5) and cultured in infection medium with cycloheximide (CHX). At 6 h p.i. cRNA $(\mathrm{H})$ or vRNA levels of the NA segment (I) were assessed using strand specific RT-PCR and are shown as mean $n$-fold of WT ( \pm SEM), $n=3$. Statistical significance was assessed using a multiple comparison one-way ANOVA-test ( ${ }^{*} p \leq 0.05$; $\left.{ }^{* * *} p \leq 0.001 ;{ }^{* * *} p \leq 0.0001\right)$. K: NP binding to the trimeric polymerase assessed by co-affinity precipitation. HEK293-T cells were transfected with PB1, PB2, HA-tagged PA and strep-tagged NP. At 24 h p.t., the constituted polymerase bound by NP was strep-purified. Co-precipitated proteins were detected by western blot. Quantification of the interaction is shown below the panels. Levels of HA-tagged PA were normalized to strep-tagged NP and reported below as the mean $n$-fold of WT, $n=3$. L:

Polymerase dimerization assessed by co-affinity precipitation. HEK293-T cells were transfected with PB1, PB2 and both HA-tagged and strep-tagged PA. 24 h p.t. the polymerase components bound to strep-tagged PA were purified. Co-precipitated proteins were detected by western blot. Quantification of the interaction is shown below the panels. Levels of the HAtagged PA was normalized to strep-tagged PA and reported as the mean $n$-fold of WT, $n=5$.

\section{Figure 5: PB1-K578 is ubiquitinated and interacts with E72 in the PB2 N-terminus}

A: A549 cells were co-transfected with strep-tagged PB1 or PB1-K578A and HA-tagged ubiquitin. Ubiquitin-modified PB1 subunits were strep-purified using denaturing conditions and analyzed by western blot. For de-ubiquitination, the bound PB1 constructs were treated with the ubiquitin-specific peptidase USP2.

536 B and C: Illustration of the localization of PB1-K578 in the 3D structures of the WSN polymerase (vRNA-bound conformation; A) and the 3D structure of the symmetric dimer of the H3N2 polymerase (PDB: 6QNW; B), showing the K578-PB1 containing helix (cyan), the flexible loop in the PB2 N-terminus (yellow) harboring PB2-E72 and the helix in the PA Cterminus that participates in dimer formation Distances between PB1-K578 and PB2-E72 are 
indicated in Angstrom $(\AA)$. Amino acids involved in the dimer interface are indicated. Created with ChimeraX D: Conservation of PB1-K578 and the relative distribution of alternative amino acids in human (left panel), avian (middle panel) and swine (right panel) isolates (sequences downloaded from NCBI (PB2 and PB1: 09/15/2017, PA: 10/01/2017) presented as percentages of all analyzed sequences. E: Polymerase activity of PB1-K578A/R mutants in the polymerase reconstitution assay of IAV strains SC35M and PR8. Relative FF activities are presented as the mean percentage activity relative to SC53M or PR8 WT ( \pm SEM), $n=3$. Statistical significance was assessed using a multiple comparison one-way ANOVA-test $\left({ }^{*} p \leq\right.$ $\left.0.05 ;{ }^{* * *} p \leq 0.001\right)$.

Figure 6: Interaction between PB1-K578 and PB2-E72 is dynamic and charge-dependent

A: Illustration of the structure surrounding PB1-K578 and PB2-E72. The type of secondary structures is indicated. The effect of introducing PB1-K578A/R mutations on secondary structures in distal regions of the PB2 loop (region a: AA 63-67; region b: AA 78-82) was analyzed using molecular dynamic simulations (YASARA) for $100 \mathrm{~ns}$ (table below: arrows indicate differences in secondary structure abundancies). B-D: Local molecular dynamic simulations of the interaction between position PB1-578 and PB2-72 (B) as well as PB1-578 and PB2-Q73 (C) and PB2-R101 (D) in dependency of the depicted mutations using YASARA. E: Schematic model for the role of ubiquitination at PB1-K578 during vRNA replication. Incoming vRNPs perform viral mRNA transcription leading to the expression of viral proteins including the viral polymerase proteins and NP. A fraction of the newly synthesized free trimeric polymerase complexes acquires ubiquitination at PB1-K578 (violet polymerase complexes with UB in orange), which disrupts the interaction between PB1-K578 and PB2-E72 and prevents NP-binding and early formation of the symmetric dimer. The PB1-K578 ubiquitinated polymerase interacts with the vRNP bound polymerase to form the ANP32A-stabilized asymmetric dimer. This induces the transition into the replicase conformation and stabilizes the cRNA during encapsidation. For initiation of vRNA synthesis from the cRNA template, a non-ubiquitinated polymerase (turquoise polymerase complexes) interacts with the polymerase in the cRNP by formation of the symmetric dimer. Whether the ubiquitin remains linked to the polymerase or is removed by a cellular deubiquitinating enzyme during vRNA synthesis remains unresolved.

572 


\section{Table 1: Modified lysines in the IAV polymerase}

576 List of the positions of all K- - -GG bound lysines in the PB2, PA and PB1 subunits detected in 5775 independent replicates (Rep.) at $5 \mathrm{~h} \mathrm{p}$. i. together with their location in functional domains. 578 The number of replicates in which the site was detected is indicated (Rep. of 5). Surface 579 exposure of the identified sites was assessed using 3D structural models of a WSN-adapted 580 IAV polymerase monomer bound to either VRNA or CRNA as well as the 3D structures of the 581 symmetric dimer of the H3N2 polymerase (PDB: 6QNW) and the ANP32A-stabilized 582 asymmetric dimer of the influenza $\mathrm{C}$ virus polymerase. Surface exposure is indicated with (+), 583 concealed location is indicated with (-). In the asymmetric dimer, surface exposure is also 584 itemized regarding location in the replicating (Rep) or encapsidating (Enc) polymerase.

585 Success or failure to generate recombinant viruses with the plasmids encoding the respective 586 mutations after three independent rescue attempts is encoded by $(+)=$ generation of virus, $(-)$ $587=$ no virus after three attempts, $(R)=$ generation of virus but reversion to wild type, $(S P)=$ 588 generation of virus but small plaque phenotype, $(\mathrm{LT})=$ generation of virus but low rescue titer. 589 Studies that have previously described or functionally characterized the detected lysines are 590 listed in the (References) column.

\begin{tabular}{|c|c|c|c|c|c|c|c|c|c|}
\hline \multicolumn{10}{|c|}{ PB2 } \\
\hline \multirow{2}{*}{ Domain } & \multirow{2}{*}{$\begin{array}{r}\mathrm{K}-\varepsilon-G G \\
\text { position }\end{array}$} & \multirow{2}{*}{$\begin{array}{l}\text { Rep. } \\
\text { of } 5\end{array}$} & \multicolumn{2}{|c|}{ Exposed in monomer } & \multicolumn{2}{|c|}{ Exposed in dimer } & \multicolumn{2}{|c|}{ Rec. virus } & \multirow{2}{*}{ References } \\
\hline & & & vRNA & cRNA & symmetric & asymmetric & Ala & Arg & \\
\hline \multirow{11}{*}{ 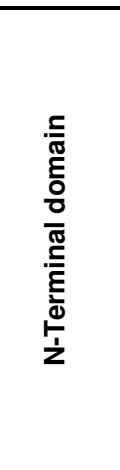 } & 32 & 2 & - & - & - & - & & & 54 \\
\hline & 41 & 4 & - & - & + & - & & & \\
\hline & 61 & 4 & + & + & + & + & & & \\
\hline & 80 & 4 & + & + & + & + & & & \\
\hline & 116 & 4 & + & + & + & + & & & \\
\hline & 121 & 4 & - & - & - & Rep - | Enc + & & & \\
\hline & 126 & 4 & + & + & + & + & & & \\
\hline & 157 & 2 & + & + & - & - & + & + & \\
\hline & 187 & 4 & + & + & + & - & & & \\
\hline & 189 & 4 & + & + & - & - & & & \\
\hline & 197 & 2 & + & + & + & + & & & \\
\hline \multirow{3}{*}{ 苘爱㐫 } & 312 & 2 & + & - & - & - & & & \\
\hline & 353 & 2 & + & + & + & + & & & 30 \\
\hline & 482 & 4 & + & + & + & Rep + | Enc - & + & $\mathrm{SP}$ & 22 \\
\hline \multirow{3}{*}{ సู่ } & 586 & 2 & + & + & + & + & & & 55 \\
\hline & 617 & 2 & - & + & - & - & - & - & \\
\hline & 670 & 2 & - & + & + & Rep - | Enc + & & & 56 \\
\hline \multirow{3}{*}{ 몰 } & 699 & 3 & + & - & + & + & + & - & 43 \\
\hline & 718 & 2 & + & + & + & - & $\mathrm{SP}$ & + & 32 \\
\hline & 752 & 4 & + & + & + & + & & & $22,32,33$ \\
\hline
\end{tabular}




\begin{tabular}{|c|c|c|c|c|c|c|c|c|c|}
\hline \multicolumn{10}{|c|}{$\mathbf{P A}$} \\
\hline \multirow{2}{*}{ Domain } & \multirow{2}{*}{$\begin{array}{l}\text { K- } \varepsilon-G G \\
\text { position }\end{array}$} & \multirow{2}{*}{$\begin{array}{l}\text { Rep. } \\
\text { of } 5\end{array}$} & \multicolumn{2}{|c|}{ Exposed in monomer } & \multicolumn{2}{|c|}{ Exposed in dimer } & \multicolumn{2}{|c|}{ Rec. virus } & \multirow{2}{*}{ References } \\
\hline & & & vRNA & cRNA & symmetric & asymmetric & Ala & Arg & \\
\hline \multirow{7}{*}{ 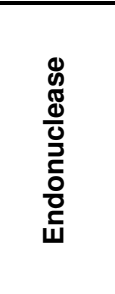 } & 22 & 4 & + & + & + & + & + & SP & 57 \\
\hline & 29 & 5 & + & + & + & + & & & \\
\hline & 102 & 2 & + & + & + & + & & & 34,36 \\
\hline & 104 & 1 & + & + & + & + & & & 34,36 \\
\hline & $113^{a}$ & 2 & + & + & + & + & & & 34 \\
\hline & 134 & 4 & - & - & - & + & - & - & $34,35,58,59$ \\
\hline & 158 & 1 & + & - & + & - & & & 35 \\
\hline \multirow{3}{*}{ 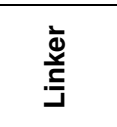 } & 213 & 3 & + & + & + & + & & & 34 \\
\hline & 245 & 3 & + & + & + & + & & & \\
\hline & 251 & 4 & + & + & + & + & & & \\
\hline \multirow{13}{*}{ 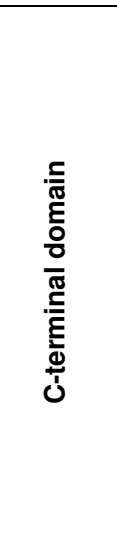 } & 262 & 4 & + & + & + & + & & & \\
\hline & 281 & 2 & - & - & - & - & & & $28,56,60,61$ \\
\hline & 309 & 2 & + & + & - & Rep + | Enc - & & & \\
\hline & 339 & 2 & + & + & + & Rep + | Enc - & & & 9,34 \\
\hline & 353 & 1 & + & + & + & + & & & 11,12 \\
\hline & 391 & 1 & + & + & + & + & & & \\
\hline & 536 & 4 & + & + & + & Rep + | Enc - & $\mathrm{R} / \mathrm{LT}$ & $\mathrm{R}$ & 39 \\
\hline & 605 & 4 & + & + & + & Rep + | Enc - & SP & + & \\
\hline & 609 & 2 & + & + & + & + & & & \\
\hline & 615 & 2 & + & + & + & Rep + | Enc - & - & + & 62 \\
\hline & 626 & 3 & + & + & + & + & & & 63 \\
\hline & 635 & 5 & + & + & + & + & - & SP/LT & 37 \\
\hline & 643 & 3 & - & - & - & - & & & 61,63 \\
\hline \multicolumn{10}{|c|}{ PB1 } \\
\hline \multirow{2}{*}{ Domain } & \multirow{2}{*}{$\begin{array}{r}\mathrm{K}-\varepsilon-\mathrm{GG} \\
\text { position }\end{array}$} & \multirow{2}{*}{$\begin{array}{l}\text { Rep. } \\
\text { of } 5\end{array}$} & \multicolumn{2}{|c|}{ Exposed in monomer } & \multicolumn{2}{|c|}{ Exposed in dimer } & \multicolumn{2}{|c|}{ Rec. virus } & \multirow{2}{*}{ References } \\
\hline & & & vRNA & cRNA & symmetric & asymmetric & Ala & Arg & \\
\hline \multirow{2}{*}{ ญे } & 121 & 3 & - & - & - & - & & & \\
\hline & 229 & 4 & - & - & - & - & - & - & $41,56,64-66$ \\
\hline \multirow{4}{*}{ 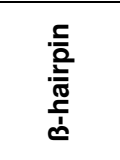 } & 347 & 2 & - & - & - & - & - & - & \\
\hline & 353 & 2 & - & - & + & + & & & 41 \\
\hline & 360 & 2 & + & + & + & + & + & LT & \\
\hline & 379 & 2 & + & + & + & + & & & \\
\hline \multirow{2}{*}{$\frac{E}{\tilde{N}}$} & 471 & 1 & - & - & - & - & - & SP & 67 \\
\hline & 480 & 3 & + & + & + & + & & & $28,41,68$ \\
\hline \multirow{4}{*}{ 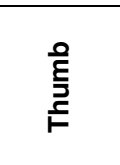 } & 578 & 2 & + & + & + & + & + & $R$ & \\
\hline & 586 & 1 & + & + & + & + & & & \\
\hline & 635 & 1 & - & - & + & - & + & + & \\
\hline & 669 & 2 & - & - & - & + & & & \\
\hline $\bar{x}$ & 736 & 2 & + & + & + & + & & & \\
\hline ப் & 737 & 4 & + & + & + & + & & & \\
\hline
\end{tabular}

Following reanalysis of the MS data additional modified sites in PB2 (K113, K627) and PB1 (K278) were detected.

${ }^{\mathrm{a} O n e}$ site in PA (K113), that was identified in previous analyses, was excluded due to treshold adjustments. 


\section{Cells}

Madin-Darby Canine Kidney cells type II (MDCK-II), human embryonic kidney 293T cells (HEK293-T) and human alveolar epithelial cells (A549) were maintained in Dulbecco's modified Eagle Medium (DMEM), supplemented with 10\% fetal calf serum (FCS; Capricorn, Germany) and 1\% Penicillin/Streptomycin (P/S; Sigma, Germany). All cells were cultured at $37^{\circ} \mathrm{C}$ and $5 \% \mathrm{CO}_{2}$. MDCK-II, HEK293-T and A549 cells were provided by Martin Schwemmle (Freiburg, Germany).

602

603

\section{Plasmids}

604

605

For generation of recombinant influenza $A$ viruses the eight reverse genetics bidirectional 606 pHW-2000 plasmids encoding vRNA and viral proteins derived from the A/WSN/1933 (H1N1) (WSN) virus were used ${ }^{69}$. Mutations in pHW-2000 PB2, PB1 and PA plasmids were introduced by site-directed mutagenesis (SDM). To create overexpressing plasmids encoding for mutated PB2, PB1 and PA of WSN, A/PR/8/1934 (PR8) and A/SC35M/1980 (SC35M), the respective pHW-2000 plasmids bearing the respective mutations were used as templates and subjected to assembly PCR to introduce restriction sites for Notl and Xhol restriction enzymes (NEB, USA). The resulted PCR fragments were cloned into PCAGGs plasmids. pCAGGs vectors that encode Strep-tagged polymerase subunit constructs of WSN-derived wild type PB2, PB1 and PA were created likewise. The PCAGGs plasmids encoding C-terminally HA-tagged PB1 and PA fusion proteins were a kind gift from Martin Schwemmle (Freiburg, Germany) and described previously ${ }^{70}$. The pPoll-Firefly reporter plasmid encodes a minus-sensed luciferase gene flanked by the 5' and 3' non-coding regions of the NS segment. Likewise, the pPoll-Firefly-UPPromoter encodes a plus-sensed luciferase gene and has been described before ${ }^{71}$. Both were used for minigenome assays together with the pTK-Renilla plasmid. pRK5-HA-Ubiquitin-WT was a gift from Ted Dawson (Addgene plasmid \#17608) and pcDNA3-HA-NEDD8 was a gift from Edward Yeh (Addgene plasmid \#18711). The pCMV2b-RGS-His-ISG15 WT plasmid was a gift from Gerrit Praefcke (Langen, Germany).

624 For detection of ubiquitination, A549 cells were cotransfected with pCAGGs plasmids expressing nStrep-PB2-, nStrep-PB1 or nStrep-PA fusion proteins with HA-Ubiquitin using XtremeGENE HP DNA Transfection Reagent (Sigma, Germany). Due to the reduced expression levels of plasmids that encode HA-tagged NEDD8 or His-tagged ISG15, the modification of the polymerase subunits with these UBLs was detected in HEK293-T cells by co-transfection of the respective plasmids using Lipofectamin 2000 transfection reagent (Invitrogen, USA). A549 cells were processed $48 \mathrm{~h}$ post transfection (p. t.) and HEK293-T cells 24 as previously described ${ }^{72}$. Briefly, cells were treated with a lysis buffer (2\% SDS, $150 \mathrm{mM} \mathrm{NaCl}, 2 \mathrm{mM}$ 
$10 \mathrm{~min}$, sonicated for $30 \mathrm{~s}$, renatured in a dilution buffer (10 mM TRIS HCl (pH 8.0), $150 \mathrm{mM}$ $\mathrm{NaCl}, 2 \mathrm{mM}$ EDTA, $1 \%$ Triton $\mathrm{X}-100)$ for $1 \mathrm{~h}$ at $4{ }^{\circ} \mathrm{C}$ and then clarified by centrifugation. Clarified lysates were adjusted to equal amounts using Pierce BCA assay (Thermo Fisher, USA) and precipitated using Strep-Tactin sepharose beads (IBA Lifescience, USA) at $4{ }^{\circ} \mathrm{C}$ for $2 \mathrm{~h}$. Beads were washed thrice using a stringent washing buffer $(10 \mathrm{mM} \mathrm{TRIS-HCl}(\mathrm{pH} \mathrm{8.0})$, $1 \mathrm{M} \mathrm{NaCl}, 1 \mathrm{mM}$ EDTA, 1\% Igepal CA-630). In case of deubiquitinase treatment, the beads were washed twice with DUB reaction buffer (1x), as provided in UbiCREST Kit ${ }^{73}$ and incubated with USP2 at $37^{\circ} \mathrm{C}$ for 30 min according to manufacturer description. Precipitates proteins were eluted using $1 \times$ Laemmli buffer (0.25 M TRIS ( $\mathrm{pH} \mathrm{6.8),40 \%} \mathrm{glycerol,} 8 \%$ SDS, $10 \% \beta$-mercaptoethanol, $0.01 \%$ bromophenol blue) by incubation at $95^{\circ} \mathrm{C}$ for $5 \mathrm{~min}$. The precipitated samples were subjected to SDS-PAGE and western blot. Covalently bound modification by HA-Ubiquitin, HA-NEDD8 or His-ISG15 of each polymerase subunit was detected using primary antibodies directed against HA-Tag (clone 3F10, Roche, Germany), NEDD8 (clone Y297, Abcam, UK) or His-Tag (clone HIS.H8, Thermo Fisher, USA). Polymerase subunits were detected using anti-PB2 (GTX125926, Genetex, USA), anti-PB1 (GTX125923, Genetex, USA) and anti-PA (GTX125932, Genetex, USA) antibodies.

\section{Mass spectrometry analyses of K- $\varepsilon$-GG-enriched peptides}

Infection was carried out by incubating $10^{8}$ A549 cells with IAV WSN in PBS supplemented with $0.7 \% \mathrm{BSA}, 1 \% \mathrm{CaCl}_{2}$ and $1 \% \mathrm{MgCl}_{2}$ at a multiplicity of infection (MOI) of 20 for 30 min at $4^{\circ} \mathrm{C}$ and subsequently for additional $30 \mathrm{~min}$ at $37^{\circ} \mathrm{C}$. For the detection of the ubiquitin remnant motif (K-ع-GG) on the IAV polymerase subunits, the PTMScan®-Kit (Cell Signaling Technology, USA) was applied according to manufacturer's protocol. Briefly, $5 \mathrm{~h}$ post infection (p.i.) cells were lysed using Urea lysis buffer (20 mM HEPES (pH 8), 9 M urea, 1 mM sodium orthovanadate, $2.5 \mathrm{mM}$ sodium pyrophosphate, $1 \mathrm{mM} \beta$-glycerophosphate), followed by sonication. Clarified lysates were reduced and alkylated using dithiothreitol (DTT) and chloroacetamide, respectively, and diluted 3-fold with $20 \mathrm{mM} \mathrm{HEPES} \mathrm{(pH} \mathrm{8.0).} \mathrm{Lysates} \mathrm{were}$ trypsin digested by incubation with $1 \%$ TPCK-Trypsin $(\mathrm{m} / \mathrm{v}$ in $1 \mathrm{mM} \mathrm{HCl})$ over night at $25^{\circ} \mathrm{C}$, desalted using Sep-Pak® C18 cartridges and immunoaffinity purification (IAP) using the anti-

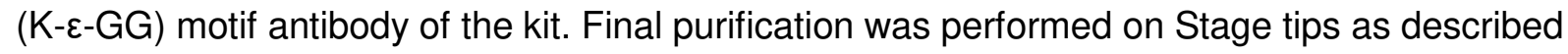
before ${ }^{74}$.

Peptides enriched for the ubiquitin remnant motif (K- $\varepsilon-\mathrm{GG}$ ) were dissolved in either $0.5 \%$ acetic acid or $0.1 \%$ formic acid prior to LC-MSMS analysis using a LTQ Velos Orbitrap $(n=1)$ or $Q$ Exactive HF mass spectrometer ( $n=3$; Thermo Scientific, USA), respectively. Each MS instrument was online coupled to an EASY nLC nanoflow HPLC system via a Nanospray Flex ion source (Thermo Scientific, USA), harboring in-house packed fused silica capillary column (length 15cm; ID $75 \mu \mathrm{m}$; ReproSil-Pur C18-AQ, $3 \mu \mathrm{m}$ ). Following loading, bound peptides were eluted using a linear 240 min gradient from $3-40 \%$ B (80\% ACN, 0.5\% acetic acid) followed 671 by a short gradient from $35-98 \% \mathrm{~B}$ in $10 \mathrm{~min}$ at a flow rate of 300 or $250 \mathrm{nl} / \mathrm{min}$. After washing 
at $98 \%$ B the column was re-equilibrated at starting conditions. The Orbitrap Velos Pro mass spectrometer was operated in the positive ion mode, switching in a data-dependent fashion between survey scans in the orbitrap (mass range $\mathrm{m} / \mathrm{z}=300-1650$; resolution $\mathrm{R}=60000$; target value $=1 \mathrm{e} 6$; lockmass set to 445.120025$)$ and collision induced fragmentation and MS/MS acquisition in the LTQ part. MS/MS spectra of the 15 most intense ion peaks detected in the MS1 scans were recorded. Conditions for the analysis using the $Q$ Exactive HF mass spectrometer were kept similar (Top17 DDA; Full MS R = 60000; AGC target = 3e6; Maximum $\mathrm{IT}=100 \mathrm{~ms}$; scan range $300-1750 \mathrm{~m} / \mathrm{z}$; ddMS2 R = 15000; $\max \mathrm{IT}=50 \mathrm{~ms}$; NCE = $27 \mathrm{~V}$ ). Dynamic exclusion was enabled on both MS systems.

Raw MS data were processed using MaxQuant (v. 2.0.1.0) with the built-in Andromeda search engine. Tandem mass spectra were searched against the Influenza A virus (strain A/WilsonSmith/1933 H1N1; UP000000834.fasta; version from 10/2016) as well as against the human UniprotKB database (UP000005640_9606.fasta; version from 04/2019) concatenated with reversed sequence versions of all entries and also containing common lab contaminants. Carbamidomethylation on cysteine residues was set as fixed modification for the search in the database, while oxidation at methionine and acetylation of the protein N-termini were set as variable modifications. For the identification of ubiquitination sites, modification of lysine residues with the remnant diglycyl motif was allowed as additional variable modification. Trypsin was defined as the digesting enzyme, allowing a maximum of two missed cleavages and requiring a minimum length of 7 amino acids. The maximum allowed mass deviation was $20 \mathrm{ppm}$ for MS and $0.5 \mathrm{Da}$ for MS/MS scans. Common lab contaminants and proteins containing reverse sequences that were derived from the decoy database were filtered out from the dataset prior to any further analysis. Protein groups were regarded as being unequivocally identified with a false discovery rate (FDR) of $1 \%$ for both the peptide and protein identifications. Ubiquitination sites were accepted when they were identified with a localization probability of $>0.75$ (class I sites). PX partial: All mass spectrometry data have been deposited to the ProteomeXchange Consortium (http://proteomecentral.proteomexchange.org) via the PRIDE partner repository with the dataset identifier PXD030816, DOI: 10.6019/PXD030816 ${ }^{75}$.

\section{Sequence analysis}

PB1, PB2 and PA sequences of human, swine and avian IAV isolates were downloaded from NCBI. 2004 sequences for IAV PB2 and 1630 sequences for IAV PB1 were downloaded on 09/15/2017. 2016 sequences for IAV PA were downloaded on 10/01/2017. The cd-hit was used to assign clusters of $99 \%$ sequence identity. Sequences of each protein were aligned using MUSCLE (multiple sequence comparison by Log-Expectation).

\section{Polymerase reconstitution assay}

709 HEK293-T cells were cotransfected with 50 ng of pCAGGs plasmids expressing PA, PB2, PB1 710 (either WT or mutant) together with $200 \mathrm{ng}$ of pCAGGs-NP plasmids and $25 \mathrm{ng}$ of both pTK- 
Renilla plasmids and the vRNA expressing pPoll-Firefly or the cRNA expressing pPoll-FireflyUp-Promoter plasmids using Lipofectamin ${ }^{\mathrm{TM}} 2000.24 \mathrm{~h}$ post transfection cells were lysed and relative polymerase activity was examined using the Dual-Luciferase Reporter Assay (Promega) and reporter gene expression was determined by measuring luminescence using a MicroLumat Plus LB96V luminometer (Berthold Technologies, Germany).

\section{Generation of recombinant viruses}

718 For generating recombinant viruses co-cultivated HEK293-T cells and MDCK-II cells were co719 transfected with the eight pHW-2000 plasmids derived from WSN (WT or mutant) using Lipofectamin $^{\mathrm{TM}} 2000$ (Invitrogen, USA). After $6 \mathrm{~h}$ of incubation in Gibco ${ }^{\mathrm{TM}}$ Opti-MEM ${ }^{\mathrm{TM}}$ । Reduced Serum Medium (Thermo Fisher, USA) at $37^{\circ} \mathrm{C}$ the supernatant was removed and cells were incubated with infection medium (DMEM supplemented with $0.7 \%$ BSA, $1 \%$ $\mathrm{CaCl}_{2} / \mathrm{MgCl}_{2}$ and TPCK-Trypsin) for $48 \mathrm{~h}$ at $37^{\circ} \mathrm{C}$. The virus rescue supernatant was plaque purified on MDCK-II cells by plaque assays using Oxoid ${ }^{\mathrm{TM}}$ purified agar (Thermo Fisher, USA). Then, MDCK-II cells were infected with single plaques diluted in infection PBS and incubated in infection medium for $48 \mathrm{~h}$ at $37^{\circ} \mathrm{C}$. Final virus stocks were obtained by passaging this supernatant once on MDCK-II cells with a defined MOI of 0.001 . To control for the respective mutation, MDCK-II cells were infected with an MOI of 2. After $6 \mathrm{~h}$, cells were lysed and RNA was extracted using the RNeasy Plus Mini Kit (Qiagen, Germany). The respective gene segment was amplified using OneStep RT-PCR Kit (Qiagen, Germany) and sequenced by Sanger sequencing (Eurofins Genomics, Germany).

\section{Deep sequencing using Illumina NGS}

734 Following isolation of the viral RNA from virus supernatant using QIAamp Viral RNA Mini Kit 735 (Qiagen, Germany), each vRNA segment was reverse transcribed using the SuperScript ${ }^{\mathrm{TM}}$ III 736 One-Step RT PCR System with Platinum ${ }^{\text {TM }}$ Taq DNA polymerase (Invitrogen, USA). In the following approximately $1 \mathrm{ng}$ of the pooled PCR products was used as template and introduced into library preparation with the Nextera XT DNA Sample Preparation Kit (Illumina, San Diego, CA, USA) and paired-end sequenced with the 2x250 bp MiSeq Reagent Kit v2 (Illumina) with an average insert size of $300 \mathrm{bp}$ on a MiSeq instrument (Illumina). The protocols for library preparation and sequencing were conducted as recommended by the manufacturer (Illumina). After automatic demultiplexing on the MiSeq instrument, the resulting fastq files were mapped onto the Influenza A virus strain A/WSN/1933(H1N1) reference sequence (GenBank accession numbers CY034132-CY034139) using the BWA mapping algorithm implemented in the Ridom SeqSphere ${ }^{+}$software version 7 (Ridom $\mathrm{GmbH}$, Münster, Germany) with default parameters. checked manually and the variant read frequencies and percentage of each nucleotide at the respective position were determined. 
To assess the accumulation of viral segment during virus rescue with limited virus propagation HEK293-T cells were seeded solely and cotransfected with the eight pHW-2000 plasmid system derived from WSN using Lipofectamin ${ }^{\text {TM }} 2000.16 \mathrm{~h}$ and $48 \mathrm{~h}$ post transfection RNA was isolated using TRIzol (Thermo Fisher, USA). Residual DNA was digested using recombinant DNase I (Roche, Germany). The RNA was reverse transcribed with oligo(dT) or Uni12 primers using RevertAid H Minus Reverse Transcriptase (Thermo Fisher Scientific, USA). Briefly, primers and $500 \mathrm{ng}$ RNA were heated at $70^{\circ} \mathrm{C}$ for $5 \mathrm{~min}$, immediately chilled on ice for $1 \mathrm{~min}$ and then heated at $37^{\circ} \mathrm{C}$ for $2 \mathrm{~min}$. Then, a mixture of First Strand Buffer (5x, Invitrogen, USA), dNTPs and RevertAid $\mathrm{H}$ Minus Reverse Transcriptase (Thermo Fisher Scientific, USA) was added and incubated at $37^{\circ} \mathrm{C}$ for $10 \mathrm{~min}$, followed by an incubation at $42^{\circ} \mathrm{C}$ for $60 \mathrm{~min}$. Reverse transcription was terminated by incubation at $85^{\circ} \mathrm{C}$ for $5 \mathrm{~min}$. CT values were measured in duplicates using a LightCycler ${ }^{\circledR} 480$ II (Roche, Germany) and analyzed using the $2-\Delta \Delta C T$ method as described elsewhere ${ }^{76}$.

\section{Quantification of viral RNA by strand-specific RT-PCR}

HEK293-T cells were transfected with $250 \mathrm{ng}$ of pCAGGs plasmids expressing wild type or mutant PB2, PB1 and PA, $1000 \mathrm{ng}$ of NP and $100 \mathrm{ng}$ of a pol-I driven minigenome firefly reporter plasmid expressing either VRNA or $\mathrm{CRNA}$, using Lipofectamin ${ }^{\text {TM }} 2000.16 \mathrm{~h}$ p.t. RNA was isolated using TRIzol (Thermo Fisher, USA) and dissolved in RNase free water. Residual DNA was digested using recombinant DNase I (Roche, Germany). For reverse transcription a mixture of tagged primers directed to either m-, c-, or vRNA of the firefly minigenome (designed according to Kawakami et al. ${ }^{77}$ ), oligo-dT primers and $100 \mathrm{ng}$ RNA were heated at $65^{\circ} \mathrm{C}$ for $10 \mathrm{~min}$, immediately chilled on ice for $5 \mathrm{~min}$ and then heated at $60^{\circ} \mathrm{C}$ for $5 \mathrm{~min}$. Then, a mixture of First Strand Buffer (5x, Invitrogen), 0.1 M dithiothreitol, dNTPs and Maxima H Minus Reverse transcriptase (Thermo Scientific, USA) was added and incubated at $60^{\circ} \mathrm{C}$ for $60 \mathrm{~min}$. Reverse transcription was terminated by incubation at $85^{\circ} \mathrm{C}$ for $5 \mathrm{~min}$. RT-PCR was carried out using Brilliant III SYBR Green (Agilent) and primers directed to the respective tags. CT values were measured in duplicates using a LightCycler® 480 II (Roche, Germany), normalized to the expression data of the housekeeping gene glyceraldehyde 3-phosphate dehydrogenase (GADPH) and analyzed using the $2-\triangle \Delta C T$ method.

\section{cRNP stabilization assay}

783 HEK293-T cells were cotransfected with pCAGGs plasmids that express for PB2, PA and NP 784 together with pCAGGs plasmids for either wild type or mutant PB1 using Lipofectamin ${ }^{\text {TM }} 2000$. After maintaining the cells on Gibco ${ }^{\mathrm{TM}}$ Opti-MEM ${ }^{\mathrm{TM}}$ (Thermo Fisher, USA) for $6 \mathrm{~h}$, the medium was exchanged to DMEM (supplemented with 10\% FBS and 1\% P/S). $24 \mathrm{~h}$ p.t. the cells were infected with WSN wild type virus at an MOI of 5 and maintained on infection medium 788 (supplemented with $100 \mu \mathrm{g} / \mathrm{ml}$ cycloheximide $(\mathrm{CHX})$ ). For controls, PA was substituted with an 
empty vector, cells were mock infected or $\mathrm{CHX}$ was omitted. $6 \mathrm{~h}$ p.i., RNA was isolated using TRIzol (Thermo Fisher, USA). For reverse transcription the protocol of strand-specific qPCR (as described above) was applied using tagged oligo-dT primers as well as primers for c- and vRNA of the NA segment. CT values were measured in duplicates using a LightCycler ${ }^{\circledR} 480$ II (Roche, Germany), normalized to the expression data of the housekeeping gene GADPH and analyzed using the $2-\Delta \Delta C T$ method.

\section{Immunofluorescence analysis}

A549 cells were seeded on glass coverslips and transfected with pCAGGs plasmids 798 expressing either WT or mutants of PB2, PB1 (in combination with nHA-PA) and PA (in combination with $n H A-P B 1$ ) using XtremeGene ${ }^{T M}$ (Roche, Germany) 24 h p.t., cells were fixed with $3.7 \%$ formaldehyde, permeabilized with $0.1 \%$ Triton X-100 and blocked with $3 \%$ BSA in PBS. For immunostaining coverslips were incubated with primary antibodies against PB2 (Genetex, USA), PB1 (Genetex, USA), PA (Genetex, USA), HA-Tag (Roche, Germany), NP (clone GA2B, BioRad, USA), or M2 (GTX125951, Genetex, USA) overnight at $4^{\circ} \mathrm{C}$ and with secondary antibodies anti-rabbit Alexa Fluor 488 (Invitrogen, USA) or anti-rabbit Alexa Fluor 568 (Invitrogen, USA) and anti-rat Alexa Fluor 488 (Invitrogen, USA) for $1 \mathrm{~h}$ at room temperature. Cell nuclei were stained with DAPI (Thermo Fisher Scientific, USA) for 20 min at room temperature. Coverslips were mounted Mounting Medium S3023 (Dako Omnis, USA) and examined using an LSM-800 Airyscan confocal microscope (Carl Zeiss, Germany).

\section{Viral protein expression assays}

811 For assessing the viral protein expression during a single infection cycle A549 cells were 812 incubated with infection PBS at an $\mathrm{MOI}$ of 5 , at $4^{\circ} \mathrm{C}$ for $30 \mathrm{~min}$, followed by an incubation at $81337^{\circ} \mathrm{C}$ for $7 \mathrm{~min}$. Following 3 washing steps with infection PBS, the cells were then maintained 814 on infection medium at $37^{\circ} \mathrm{C}$ for $8 \mathrm{~h}$. For multi cycle approaches A549 cells were incubated with infection PBS at the indicated $\mathrm{MOI}$ at $37^{\circ} \mathrm{C}$ for $30 \mathrm{~min}$ and were then maintained on infection medium (supplemented with TPCK-Trypsin) at $37^{\circ} \mathrm{C}$ for $24 \mathrm{~h}$. At the respective time point, cells were lysed using ice cold radioimmunoprecipitation assay (RIPA) buffer (25 mM TRIS ( $\mathrm{pH} 7.5$ ), $150 \mathrm{mM} \mathrm{NaCl}, 0.1 \%$ SDS, $0.5 \%$ sodium deoxycholate, $1 \%$ Triton $\mathrm{X}-100$ ) supplemented with a protease inhibitor cocktail and clarified protein lysates were analyzed on western blot.

\section{Hemagglutination assay}

823 To quantify the total amount of virus particles after infection, the volume of the assessed supernatants was adjusted to a final volume of $50 \mu \mathrm{l}$ based on the PFU titer using infection PBS. The virus samples were serially diluted (1:2) in infection PBS until dilution 1:1024 in a Vbottom-shaped microtiter plate. Then, $50 \mu \mathrm{l}$ of fresh human erythrocytes (blood type: 0 ) was added to each well, mixed with the virus dilutions and incubated for 1 hour on $4^{\circ} \mathrm{C}$. 


\section{Affinity precipitation of NP-polymerase complexes}

830 HEK293-T cells were transfected with pCAGGs plasmids encoding for Strep-tagged NP fusion 831 proteins as well as PB2, nHA-PA and PB1 (wild type or mutant) using Lipofectamin® 2000. 832 For negative controls, NP-nStrep, PB2 and nHA-PA plasmids were substituted with an empty 833 vector. $24 \mathrm{~h}$ p.t. the cells were lysed using ice cold Co-IP lysis buffer (50 mM TRIS (pH 8.0), $150 \mathrm{mM} \mathrm{NaCl}, 5 \mathrm{mM}$ EDTA,1\% (v/v) Igepal CA-630, protease inhibitor cocktail), as described before ${ }^{78}$. Clarified cell lysates were adjusted to equal amounts using Pierce ${ }^{\mathrm{TM}}$ BCA assay (Thermo Fisher, USA) and precipitated using Strep-Tactin sepharose beads (IBA Lifescience, USA) for $2 \mathrm{~h}$ at $4^{\circ} \mathrm{C}$. Beads were washed 5 times using Co-IP washing buffer (50 mM TRIS (pH 8.0), $500 \mathrm{mM} \mathrm{NaCl}, 5 \mathrm{mM}$ EDTA, 1\% (v/v) Igepal CA-630). Precipitated proteins were eluted in Laemmli buffer (1X) and subjected to SDS-PAGE and western blotting. Coprecipitated proteins were detected using antibodies directed against Strep-Tag (clone GT661, Sigma Aldrich, Germany), HA-Tag (clone: 3F10, Roche, Germany), PB1 (Genetex, USA), PB2 (Genetex, USA) and Tubulin (clone DM1A, Sigma Aldrich, Germany).

\section{Dimerization of the viral polymerase}

HEK293-T cells were transfected with pCAGGs plasmids expressing PA-nStrep, PA-nHA, wild type or mutant PB1 and PB2, using Lipofectamin ${ }^{\mathrm{TM}} 2000$ (Invitrogen, USA). For negative controls PA-nStrep, PA-nHA and PB2 plasmids were substituted with an empty vector. After $24 \mathrm{~h}$ cells were lysed in $400 \mu \mathrm{l}$ of TRIS lysis buffer (50 mM TRIS-HCl (pH 7.6), $200 \mathrm{mM} \mathrm{NaCl}$, $25 \%$ glycerol, $0.5 \%$ Igepal CA-630, $1 \mathrm{mM} \mathrm{DTT}$, protease inhibitor cocktail) for 30 min at $4^{\circ} \mathrm{C}$, as described before ${ }^{12}$. Clarified cell lysates were adjusted to equal amounts using Pierce ${ }^{\mathrm{TM}}$ BCA assay (Thermo Fisher, USA) and precipitated using Strep-Tactin sepharose beads (IBA Lifescience, USA) at $4^{\circ} \mathrm{C}$ for $16 \mathrm{~h}$. Beads were washed 5 times with a washing buffer $(10 \mathrm{mM}$ TRIS-HCl (pH 7.6), $150 \mathrm{mM} \mathrm{NaCl}, 10 \%$ glycerol, 0.1\% lgepal CA-630) and proteins were eluted using $1 \mathrm{x}$ Laemmli buffer. Precipitated proteins were detected by western blot using antibodies directed against Strep-Tag (clone GT661, Sigma Aldrich, Germany), HA-Tag (clone: 3F10, Roche, Germany), PB1 (Genetex, USA) and Tubulin (clone DM1A, Sigma Aldrich, Germany).

\section{Homology modeling of WSN polymerase structures}

For both models of IAV WSN polymerase bound to VRNA as well as the IAV WSN polymerase model bound to cRNA comparative homology modeling was performed using MODELLER (https://www.salilab.org/modeller). For the vRNA bound polymerase model the crystal structure of the heterotrimeric Bat Influenza A polymerase bound to the vRNA promoter (PDB: 4WSB) served as a template, for the cRNA bound polymerase model the crystal structure of Influenza B virus in complex with 5' cRNA (PDB: 5EPI) served as a template. Crystal structure sequences were aligned with the polymerase subunit sequences of WSN ((i) 
A/WSN/1933(H1N1) PA (GenBank: CY034137); (ii) A/WSN/1933(H1N1) PB1 (GenBank: CY034138 and (iii) A/WSN/1933(H1N1) PB2 (GenBank: CY034139) and 100 homology models (including loop models) were created of both conformation states based on these WSN sequences. The RNA was transferred as a rigid body from the respective original structure. A 'slow' optimization protocol and 'slow' molecular dynamics refinement was further applied. The linker regions were modeled using "loop model" without imposing any restraints on its structure. The best models were selected based on a normalized energy score. The models were scored using the normalized DOPE score available in MODELLER. Subsequently, superposition models were prepared by VMD1.9.3 (https://www-s.ks.uiuc.edu/Research/vmd/vmd-1.9.3/) and figures were generated using PyMOL (Version 2.3.0, The PyMOL Molecular Graphics System, Schrodinger LLC) ${ }^{79,80}$.

\section{Molecular dynamic simulations}

The homology model of the three-dimensional structure of the WSN polymerase complex bound to cRNA was used for further structural analyses. The residue PB1-K578 was mutated to K578A or K578R. Each model was energy minimized by the standard method in Yasara Structure (version 21.6.2) ${ }^{81}$. After short equilibration simulations ( $\left.\sim \mathrm{ns}\right)$ the models were subjected to $100 \mathrm{~ns}$ local molecular dynamic simulations. All residues in a $45 \AA$-sphere around residue PB1-K578 were mobile. The following standard settings were used: AMBER14 Force Field, particle-mesh Ewald / Poisson-Boltzmann cutoff $8 \AA$, periodic simulation cell boundary, long range coulomb forces, $2^{\star} 2.5$ fs time step, physiological settings $(0.9 \% \mathrm{NaCl}(\mathrm{pH} 7.4)$, water density 0.997$)$. The interactions of K578 with adjacent residues were analyzed. The complex interactions were analyzed in detail using Yasara ligand analyses technique, in which K578 was defined as ligand. Secondary structure analyses were carried out using Yasara Structure.

\section{References}

1. Ruigrok, R. W. \& Baudin, F. Structure of influenza virus ribonucleoprotein particles. II. Purified RNA-free influenza virus ribonucleoprotein forms structures that are indistinguishable from the intact influenza virus ribonucleoprotein particles. The Journal of general virology 76 ( Pt 4), 1009-1014; 10.1099/0022-1317-76-4-1009 (1995).

2. Compans, R. W., Content, J. \& Duesberg, P. H. Structure of the ribonucleoprotein of influenza virus. Journal of Virology 10, 795-800; 10.1128/JVI.10.4.795-800.1972 (1972).

3. Fodor, E. \& te Velthuis, Aartjan J. W. Structure and Function of the Influenza Virus Transcription and Replication Machinery. Cold Spring Harb Perspect Med 10, a038398; 10.1101/cshperspect.a038398 (2020).

4. Krischuns, T., Lukarska, M., Naffakh, N. \& Cusack, S. Influenza Virus RNA-Dependent RNA Polymerase and the Host Transcriptional Apparatus. Annual Review of Biochemistry 90, 321-348; 10.1146/annurev-biochem-072820-100645 (2021). 
5. Wandzik, J. M. et al. A Structure-Based Model for the Complete Transcription Cycle of Influenza Polymerase. Cell 181, 877-893.e21; 10.1016/j.cell.2020.03.061 (2020).

6. Beaton, A. R. \& Krug, R. M. Selected host cell capped RNA fragments prime influenza viral RNA transcription in vivo. Nucleic Acids Res 9, 4423-4436; 10.1093/nar/9.17.4423 (1981).

7. te Velthuis, Aartjan J. W. \& Fodor, E. Influenza virus RNA polymerase: insights into the mechanisms of viral RNA synthesis. Nature Reviews Microbiology 14, 479-493; 10.1038/nrmicro.2016.87 (2016).

8. Thierry, E. et al. Influenza Polymerase Can Adopt an Alternative Configuration Involving a Radical Repacking of PB2 Domains. Molecular Cell 61, 125-137; 10.1016/j.molcel.2015.11.016 (2016).

9. Carrique, L. et al. Host ANP32A mediates the assembly of the influenza virus replicase. Nature 587, 638-643; 10.1038/s41586-020-2927-z (2020).

10. Nilsson-Payant, B. E., tenOever, B. R. \& te Velthuis, A. J. The host factor ANP32A is required for influenza $A$ virus vRNA and cRNA synthesis. bioRxiv, 2021.04.30.442228; 10.1101/2021.04.30.442228 (2021).

11. Fan, H. et al. Structures of influenza A virus RNA polymerase offer insight into viral genome replication. Nature 573, 287-290; 10.1038/s41586-019-1530-7 (2019).

12. Chen, K.-Y., Santos Afonso, E. D., Enouf, V., Isel, C. \& Naffakh, N. Influenza virus polymerase subunits co-evolve to ensure proper levels of dimerization of the heterotrimer. PLoS Pathogens 15, e1008034; 10.1371/journal.ppat.1008034 (2019).

13. Dawson, A. R. \& Mehle, A. Flu's cues: Exploiting host post-translational modifications to direct the influenza virus replication cycle. PLoS Pathogens 14, e1007205; 10.1371/journal.ppat.1007205 (2018).

14. Hubel, P. et al. A protein-interaction network of interferon-stimulated genes extends the innate immune system landscape. Nat Immunol 20, 493-502; 10.1038/s41590-019-03233 (2019).

15. Dawson, A. R. et al. Phosphorylation controls RNA binding and transcription by the influenza virus polymerase. PLoS Pathogens 16, e1008841; 10.1371/journal.ppat.1008841 (2020).

16. Hutchinson, E. C. et al. Mapping the phosphoproteome of influenza A and B viruses by mass spectrometry. PLoS Pathogens 8, e1002993; 10.1371/journal.ppat.1002993 (2012).

17. Giese, S. et al. Role of influenza A virus NP acetylation on viral growth and replication. Nat Commun 8, 1259; 10.1038/s41467-017-01112-3 (2017).

18. Hatakeyama, D. et al. Influenza $A$ virus nucleoprotein is acetylated by histone acetyltransferases PCAF and GCN5. The Journal of Biological Chemistry 293, 71267138; 10.1074/jbc.RA117.001683 (2018).

19. Di Pietro, A. et al. TRIM22 inhibits influenza A virus infection by targeting the viral nucleoprotein for degradation. J Virol 87, 4523-4533; 10.1128/JVI.02548-12 (2013).

20. Fu, B. et al. TRIM32 Senses and Restricts Influenza A Virus by Ubiquitination of PB1 Polymerase. PLoS Pathogens 11, e1004960; 10.1371/journal.ppat.1004960 (2015).

21. Kirui, J., Mondal, A. \& Mehle, A. Ubiquitination Upregulates Influenza Virus Polymerase Function. Journal of Virology 90, 10906-10914; 10.1128/JVI.01829-16 (2016).

22. Karim, M. et al. Nonproteolytic K29-Linked Ubiquitination of the PB2 Replication Protein of Influenza A Viruses by Proviral Cullin 4-Based E3 Ligases. mBio 11; 10.1128/mBio.00305-20 (2020). 
23. Reich, S. et al. Structural insight into cap-snatching and RNA synthesis by influenza polymerase. Nature 516, 361-366; 10.1038/nature14009 (2014).

24. Sugiyama, K. et al. Structural insight into the essential PB1-PB2 subunit contact of the influenza virus RNA polymerase. The EMBO Journal 28, 1803-1811; 10.1038/emboj.2009.138 (2009).

25. Reuther, P., Mänz, B., Brunotte, L., Schwemmle, M. \& Wunderlich, K. Targeting of the influenza A virus polymerase PB1-PB2 interface indicates strain-specific assembly differences. Journal of Virology 85, 13298-13309; 10.1128/JVI.00868-11 (2011).

26. Tetsuya Toyoda, Djanybek M. Adyshev, Makoto Kobayashi, Akira Iwata and Akira Ishihama. Molecular Assembly of the Influenza Virus RNA Polymerase: Determination of the Subunit-Subunit Contact Sites (1996).

27. Poole, E., Elton, D., Medcalf, L. \& Digard, P. Functional domains of the influenza A virus PB2 protein: identification of NP- and PB1-binding sites. Virology 321, 120-133; 10.1016/j.virol.2003.12.022 (2004).

28. Pflug, A., Guilligay, D., Reich, S. \& Cusack, S. Structure of influenza A polymerase bound to the viral RNA promoter. Nature 516, 355-360; 10.1038/nature14008 (2014).

29. Hemerka, J. N. et al. Detection and characterization of influenza A virus PA-PB2 interaction through a bimolecular fluorescence complementation assay. Journal of Virology 83, 3944-3955; 10.1128/JVI.02300-08 (2009).

30. Szeto, W.-C., Hsia, H.-P., Tang, Y.-S. \& Shaw, P.-C. Interaction between influenza A virus nucleoprotein and PB2 cap-binding domain is mediated by RNA. PloS one 15, e0239899; 10.1371/journal.pone.0239899 (2020).

31. Hsia, H.-P. et al. Amino acid substitutions affecting aspartic acid 605 and valine 606 decrease the interaction strength between the influenza virus RNA polymerase PB2 '627' domain and the viral nucleoprotein. PloS one 13, e0191226; 10.1371/journal.pone.0191226 (2018).

32. Pumroy, R. A., Ke, S., Hart, D. J., Zachariae, U. \& Cingolani, G. Molecular determinants for nuclear import of influenza A PB2 by importin $\alpha$ isoforms 3 and 7. Structure (London, England : 1993) 23, 374-384; 10.1016/j.str.2014.11.015 (2015).

33. Tarendeau, F. et al. Structure and nuclear import function of the C-terminal domain of influenza virus polymerase PB2 subunit. Nature Structural \& Molecular Biology 14, 229233; 10.1038/nsmb1212 (2007).

34. Hara, K., Schmidt, F. I., Crow, M. \& Brownlee, G. G. Amino acid residues in the Nterminal region of the PA subunit of influenza A virus RNA polymerase play a critical role in protein stability, endonuclease activity, cap binding, and virion RNA promoter binding. Journal of Virology 80, 7789-7798; 10.1128/JVI.00600-06 (2006).

35. Yuan, $\mathrm{P}$. et al. Crystal structure of an avian influenza polymerase $\mathrm{PA}(\mathrm{N})$ reveals an endonuclease active site. Nature 458, 909-913; 10.1038/nature07720 (2009).

36. Xiao, S. et al. Magnesium-Dependent RNA Binding to the PA Endonuclease Domain of the Avian Influenza Polymerase. J. Phys. Chem. B 118, 873-889; 10.1021/jp408383g (2014).

37. Lukarska, M. et al. Structural basis of an essential interaction between influenza polymerase and Pol II CTD. Nature 541, 117-121; 10.1038/nature20594 (2017).

38. Wang, L. et al. Functional Genomics Reveals Linkers Critical for Influenza Virus Polymerase. J Virol 90, 2938-2947; 10.1128/JVI.02400-15 (2015).

39. He, X. et al. Crystal structure of the polymerase PA(C)-PB1(N) complex from an avian influenza H5N1 virus. Nature 454, 1123-1126; 10.1038/nature07120 (2008). 
1000

1001

1002

1003

1004

1005

1006

1007

1008

1009

1010

1011

1012

1013

1014

1015

1016

1017

1018

1019

1020

1021

1022

1023

1024

1025

1026

1027

1028

1029

1030

1031

1032

1033

1034

1035

1036

1037

1038

1039

1040

1041

1042

1043

1044

1045

40. Obayashi, E. et al. The structural basis for an essential subunit interaction in influenza virus RNA polymerase. Nature 454, 1127-1131; 10.1038/nature07225 (2008).

41. Kouba, T., Drncová, P. \& Cusack, S. Structural snapshots of actively transcribing influenza polymerase. Nature Structural \& Molecular Biology 26, 460-470; 10.1038/s41594-019-0232-z (2019).

42. Walker, A. P., Sharps, J. \& Fodor, E. Mutation of an Influenza Virus Polymerase 3' RNA Promoter Binding Site Inhibits Transcription Elongation. Journal of Virology 94; 10.1128/JVI.00498-20 (2020).

43. Zhang, T. et al. NEDDylation of PB2 Reduces Its Stability and Blocks the Replication of Influenza A Virus. Sci Rep 7, 43691; 10.1038/srep43691 (2017).

44. Ng, A. K.-L. et al. Influenza polymerase activity correlates with the strength of interaction between nucleoprotein and PB2 through the host-specific residue K/E627. PloS one 7, e36415; 10.1371/journal.pone.0036415 (2012).

45. Mukaigawa, J. \& Nayak, D. P. Two signals mediate nuclear localization of influenza virus (A/WSN/33) polymerase basic protein 2. Journal of Virology 65, 245-253; 10.1128/JVI.65.1.245-253.1991 (1991).

46. Jorba, N., Coloma, R. \& Ortín, J. Genetic trans-complementation establishes a new model for influenza virus RNA transcription and replication. PLoS Pathogens 5, e1000462; 10.1371/journal.ppat.1000462 (2009).

47. Vreede, F. T., Jung, T. E. \& Brownlee, G. G. Model suggesting that replication of influenza virus is regulated by stabilization of replicative intermediates. Journal of Virology 78, 9568-9572; 10.1128/JVI.78.17.9568-9572.2004 (2004).

48. Turrell, L., Lyall, J. W., Tiley, L. S., Fodor, E. \& Vreede, F. T. The role and assembly mechanism of nucleoprotein in influenza A virus ribonucleoprotein complexes. Nat Commun 4, 1591; 10.1038/ncomms2589 (2013).

49. Vreede, F. T., Ng, A. K.-L., Shaw, P.-C. \& Fodor, E. Stabilization of Influenza Virus Replication Intermediates Is Dependent on the RNA-Binding but Not the HomoOligomerization Activity of the Viral Nucleoprotein. Journal of Virology 86, 640; 10.1128/JVI.06651-11 (2012).

50. Shin, Y.-C., Chen, J.-H. \& Chang, S.-C. The molecular determinants for distinguishing between ubiquitin and NEDD8 by USP2. Sci Rep 7, 2304; 10.1038/s41598-017-02322-x (2017).

51. Lin, Y.-C., Jeng, K.-S. \& Lai, M. M. C. CNOT4-Mediated Ubiquitination of Influenza A Virus Nucleoprotein Promotes Viral RNA Replication. mBio 8; 10.1128/mBio.00597-17 (2017).

52. Biquand, E. et al. Comparative Profiling of Ubiquitin Proteasome System Interplay with Influenza A Virus PB2 Polymerase Protein Recapitulating Virus Evolution in Humans. mSphere 2; 10.1128/mSphere.00330-17 (2017).

53. Mayer, D. et al. Identification of cellular interaction partners of the influenza virus ribonucleoprotein complex and polymerase complex using proteomic-based approaches. Journal of Proteome Research 6, 672-682; 10.1021/pr060432u (2007).

54. Hulme, K. D. et al. A paucigranulocytic asthma host environment promotes the emergence of virulent influenza viral variants. eLife 10; 10.7554/eLife.61803 (2021).

55. Kirui, J., Bucci, M. D., Poole, D. S. \& Mehle, A. Conserved features of the PB2 627 domain impact influenza virus polymerase function and replication. Journal of Virology $\mathbf{8 8}$, 5977-5986; 10.1128/JVI.00508-14 (2014). 
56. Pagadala, N. S., Bhat, R., Kumar D, J. \& Landi, A. Discovery of anti-influenza nucleoside triphosphates targeting the catalytic site of $\mathrm{A} / \mathrm{PR} / 8 / 34 / \mathrm{H} 1 \mathrm{~N} 1$ polymerase. Med Chem Res 29, 1-15; 10.1007/s00044-020-02561-0 (2020).

57. Keown, J. R. et al. Mapping inhibitory sites on the RNA polymerase of the 1918 pandemic influenza virus using nanobodies. Nat Commun 13, 1-11; 10.1038/s41467-021-27950-w (2022).

58. Dias, A. et al. The cap-snatching endonuclease of influenza virus polymerase resides in the PA subunit. Nature 458, 914-918; 10.1038/nature07745 (2009).

59. Kowalinski, E. et al. Structural analysis of specific metal chelating inhibitor binding to the endonuclease domain of influenza pH1N1 (2009) polymerase. PLoS Pathogens 8, e1002831; 10.1371/journal.ppat.1002831 (2012).

60. Wu, N. C. et al. Functional Constraint Profiling of a Viral Protein Reveals Discordance of Evolutionary Conservation and Functionality. PLOS Genetics 11, e1005310; 10.1371/journal.pgen.1005310 (2015).

61. Chen, H. et al. HDAC6 Restricts Influenza A Virus by Deacetylation of the RNA Polymerase PA Subunit. J Virol 93; 10.1128/JVI.01896-18 (2019).

62. Gabriel, G. et al. The viral polymerase mediates adaptation of an avian influenza virus to a mammalian host. Proceedings of the National Academy of Sciences of the United States of America 102, 18590-18595; 10.1073/pnas.0507415102 (2005).

63. Moen, S. O. et al. Structural analysis of H1N1 and H7N9 influenza A virus PA in the absence of PB1. Sci Rep 4, 5944; 10.1038/srep05944 (2014).

64. Goldhill, D. H. et al. The mechanism of resistance to favipiravir in influenza. Proceedings of the National Academy of Sciences 115, 11613-11618; 10.1073/pnas.1811345115 (2018).

65. Li, C. et al. Integrating computational modeling and functional assays to decipher the structure-function relationship of influenza virus PB1 protein. Sci Rep 4, 7192; 10.1038/srep07192 (2014).

66. Du, Y. et al. Annotating Protein Functional Residues by Coupling High-Throughput Fitness Profile and Homologous-Structure Analysis. mBio 7; 10.1128/mBio.01801-16 (2016).

67. Dreger, M., Leung, B. W., Brownlee, G. G. \& Deng, T. A quantitative strategy to detect changes in accessibility of protein regions to chemical modification on heterodimerization. Protein Science 18, 1448-1458; 10.1002/pro.159 (2009).

68. Chu, C. et al. Functional analysis of conserved motifs in influenza virus PB1 protein. PLOS ONE 7, e36113; 10.1371/journal.pone.0036113 (2012).

69. Hoffmann, E., Neumann, G., Kawaoka, Y., Hobom, G. \& Webster, R. G. A DNA transfection system for generation of influenza $A$ virus from eight plasmids. Proceedings of the National Academy of Sciences of the United States of America 97, 6108-6113; 10.1073/pnas.100133697 (2000).

70. Mänz, B., Brunotte, L., Reuther, P. \& Schwemmle, M. Adaptive mutations in NEP compensate for defective H5N1 RNA replication in cultured human cells. Nature Communications 3, 802; 10.1038/ncomms1804 (2012).

71. Azzeh, M., Flick, R. \& Hobom, G. Functional analysis of the influenza A virus cRNA promoter and construction of an ambisense transcription system (2001).

72. Choo, Y. S. \& Zhang, Z. Detection of protein ubiquitination. Journal of Visualized Experiments; 10.3791/1293 (2009). 
73. Hospenthal, M. K., Mevissen, T. E. T. \& Komander, D. Deubiquitinase-based analysis of ubiquitin chain architecture using Ubiquitin Chain Restriction (UbiCRest). Nat Protoc 10, 349-361; 10.1038/nprot.2015.018 (2015).

74. Rappsilber, J., Ishihama, Y. \& Mann, M. Stop and go extraction tips for matrix-assisted laser desorption/ionization, nanoelectrospray, and LC/MS sample pretreatment in proteomics. Analytical chemistry 75, 663-670; 10.1021/ac026117i (2003).

75. Perez-Riverol, Y. et al. The PRIDE database and related tools and resources in 2019: improving support for quantification data. Nucleic Acids Research 47, D442-D450; 10.1093/nar/gky1106 (2019).

76. Livak, K. J. \& Schmittgen, T. D. Analysis of relative gene expression data using real-time quantitative PCR and the 2(-Delta Delta C(T)) Method. Methods 25, 402-408; 10.1006/meth.2001.1262 (2001).

77. Kawakami, E. et al. Strand-specific real-time RT-PCR for distinguishing influenza vRNA, cRNA, and mRNA. Journal of Virological Methods 173, 1-6; 10.1016/j.jviromet.2010.12.014 (2011).

78. Verhelst, J., Vlieger, D. de \& Saelens, X. Co-immunoprecipitation of the Mouse Mx1 Protein with the Influenza A Virus Nucleoprotein. Journal of Visualized Experiments; $10.3791 / 52871$ (2015).

79. Schrödinger, LLC and Warren DeLano. PyMOL ,

80. Humphrey, W., Dalke, A. \& Schulten, K. VMD: Visual molecular dynamics. Journal of molecular graphics 14, 33-38; 10.1016/0263-7855(96)00018-5 (1996).

81. Krieger, E. et al. Improving physical realism, stereochemistry, and side-chain accuracy in homology modeling: Four approaches that performed well in CASP8. Proteins 77 Suppl 9, 114-122; 10.1002/prot.22570 (2009). 

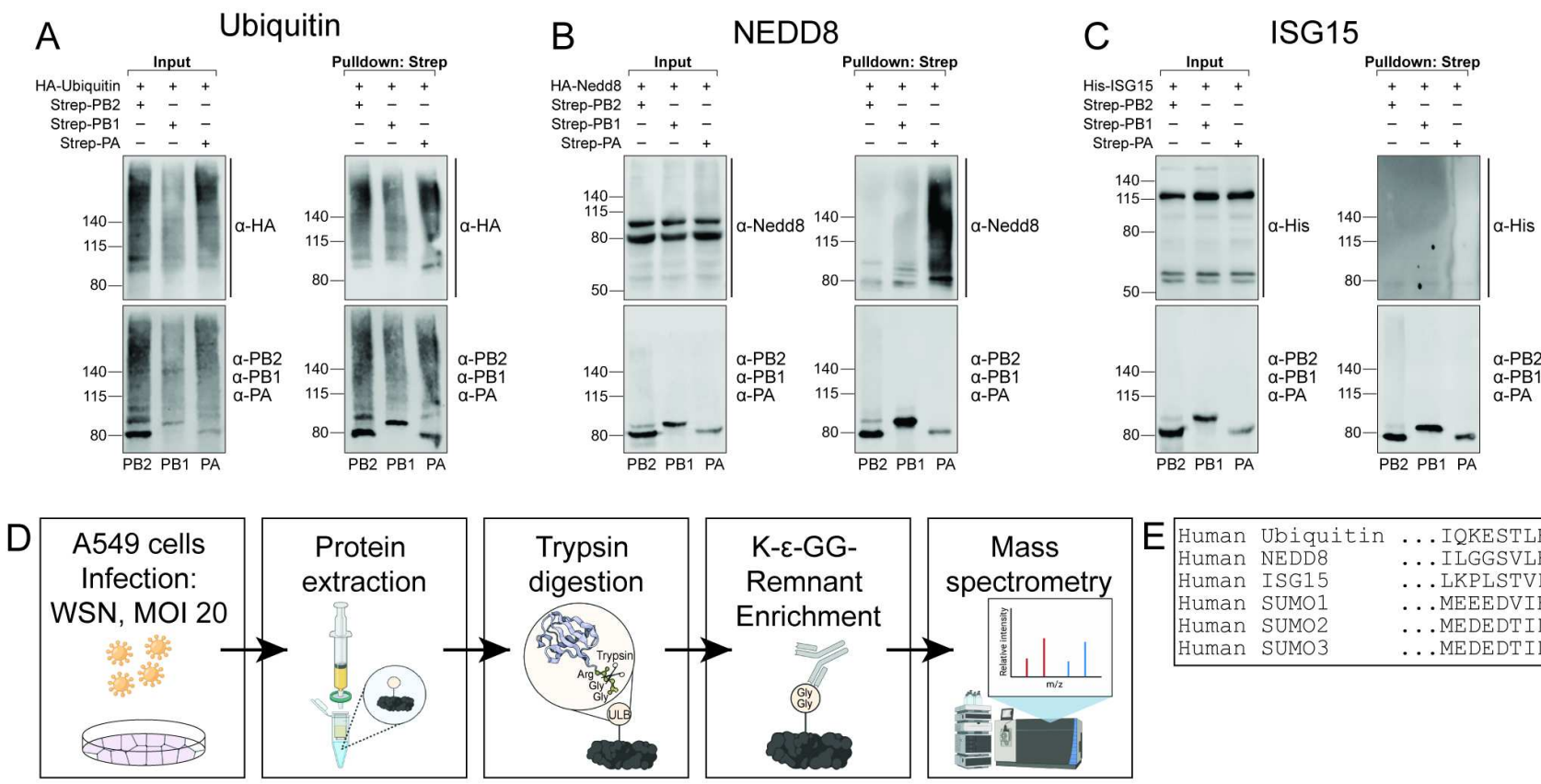

E Human Ubiquitin ... IQKESTLHLVLRIRGG Human NEDD8 ...ILGGSVLHLVLALRGG Human ISG15 ....LKPLSTVFMNLRLRGG Human SUMO1 ...MEEEDVIEVYQEQTGG Human SUMO2 Human SUMO3 .MEDEDTIDVEQOOTG QII

Conservation PB2

human

swine avian

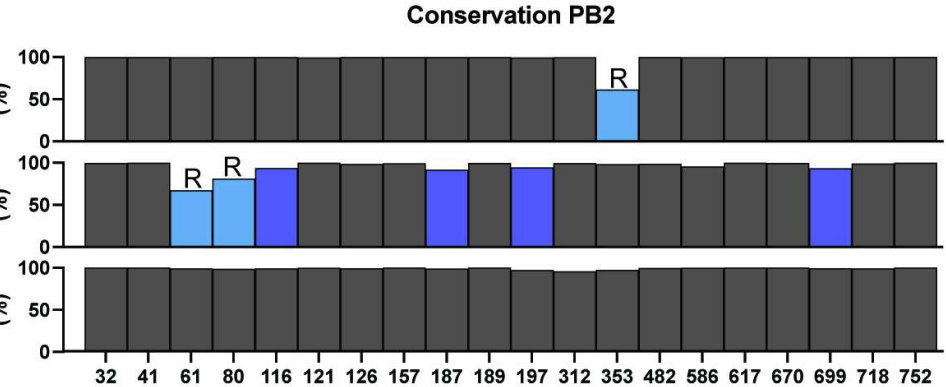

G

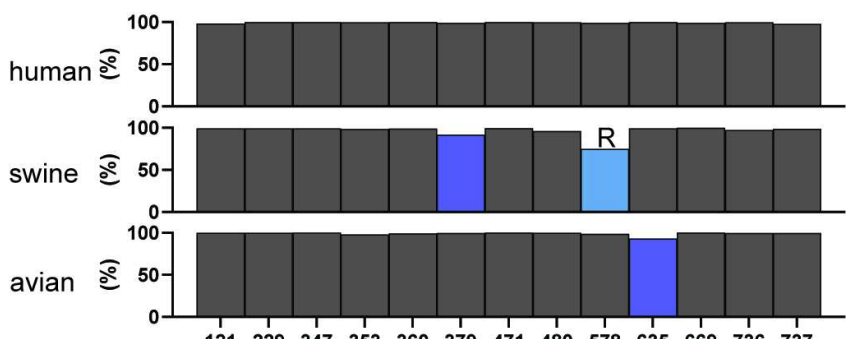

$\mathrm{H}$ human swine $\left.\begin{array}{r}100 \\ 50 \\ 0\end{array}\right]$ $\left.\begin{array}{r}100 \\ 50 \\ 0\end{array}\right]$

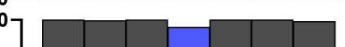
$R$ Conservation PA
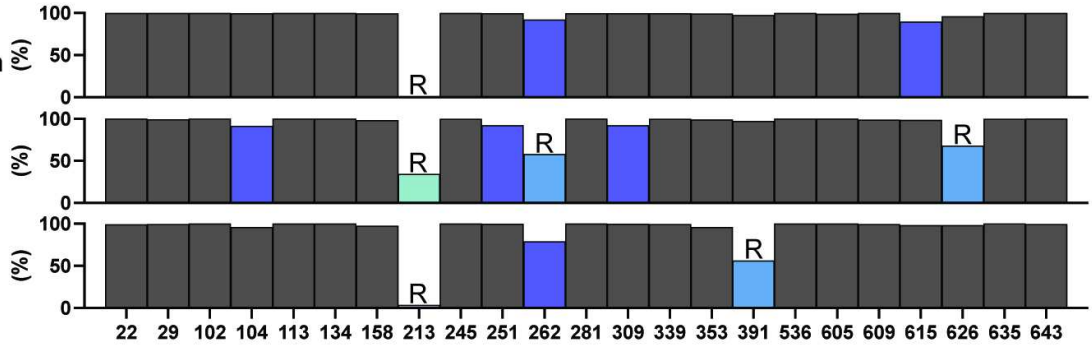
avian

$22 \quad 29102104113134158213245251262281309339353391536605609615626635643$

\section{Figure 1}

Identification of site-specific UB modifications in the IAV polymerase 
Figure 2

A
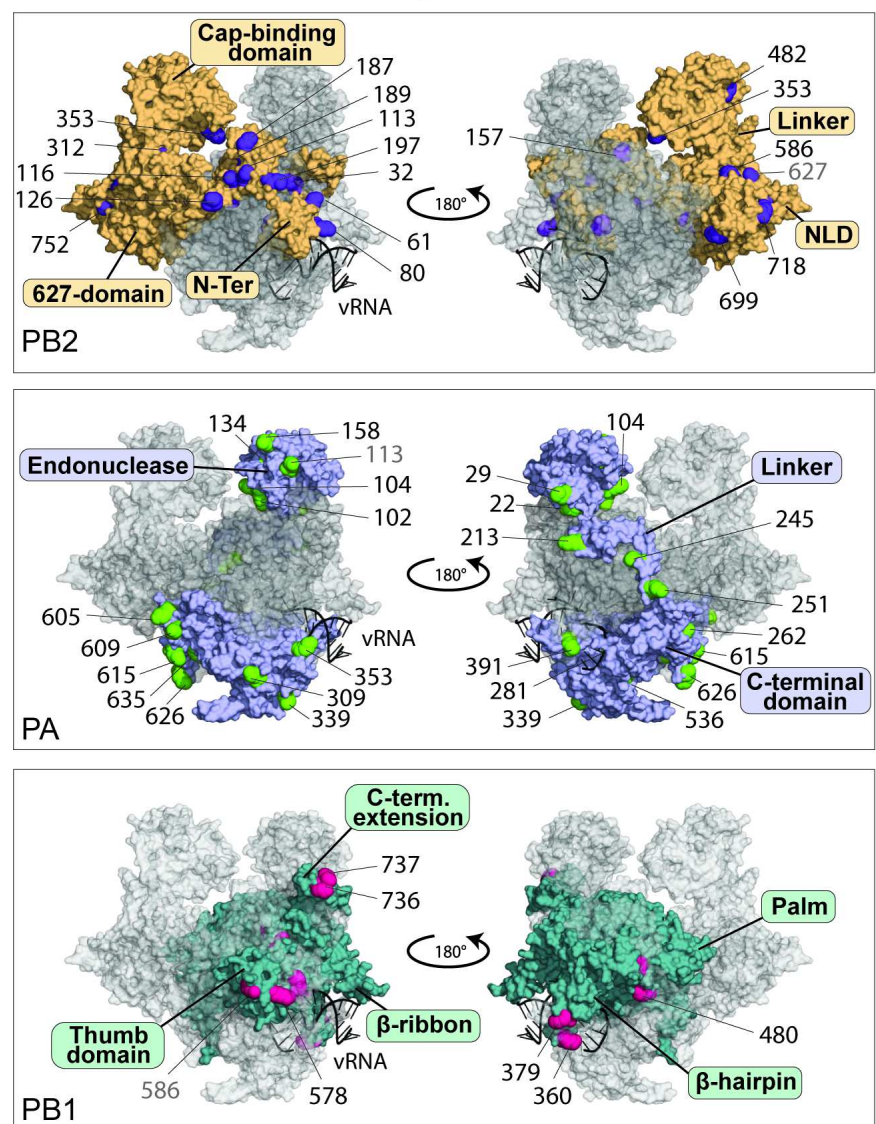

B CRNA bound polymerase structure
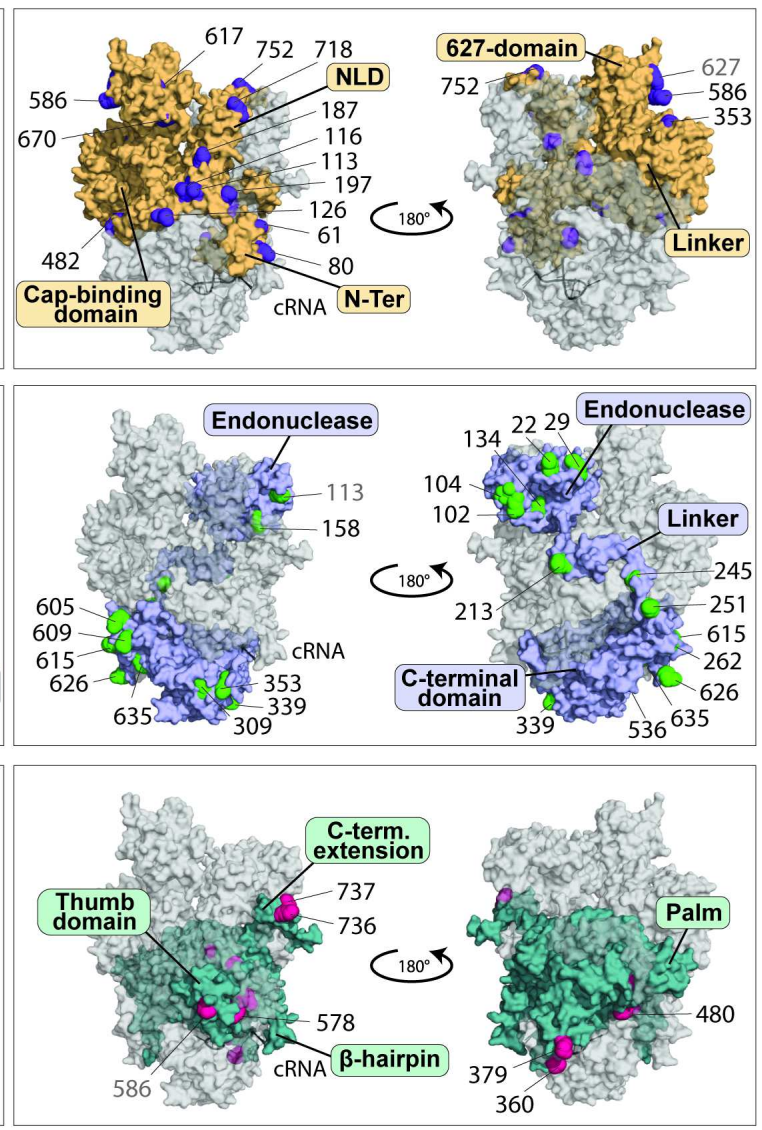

C

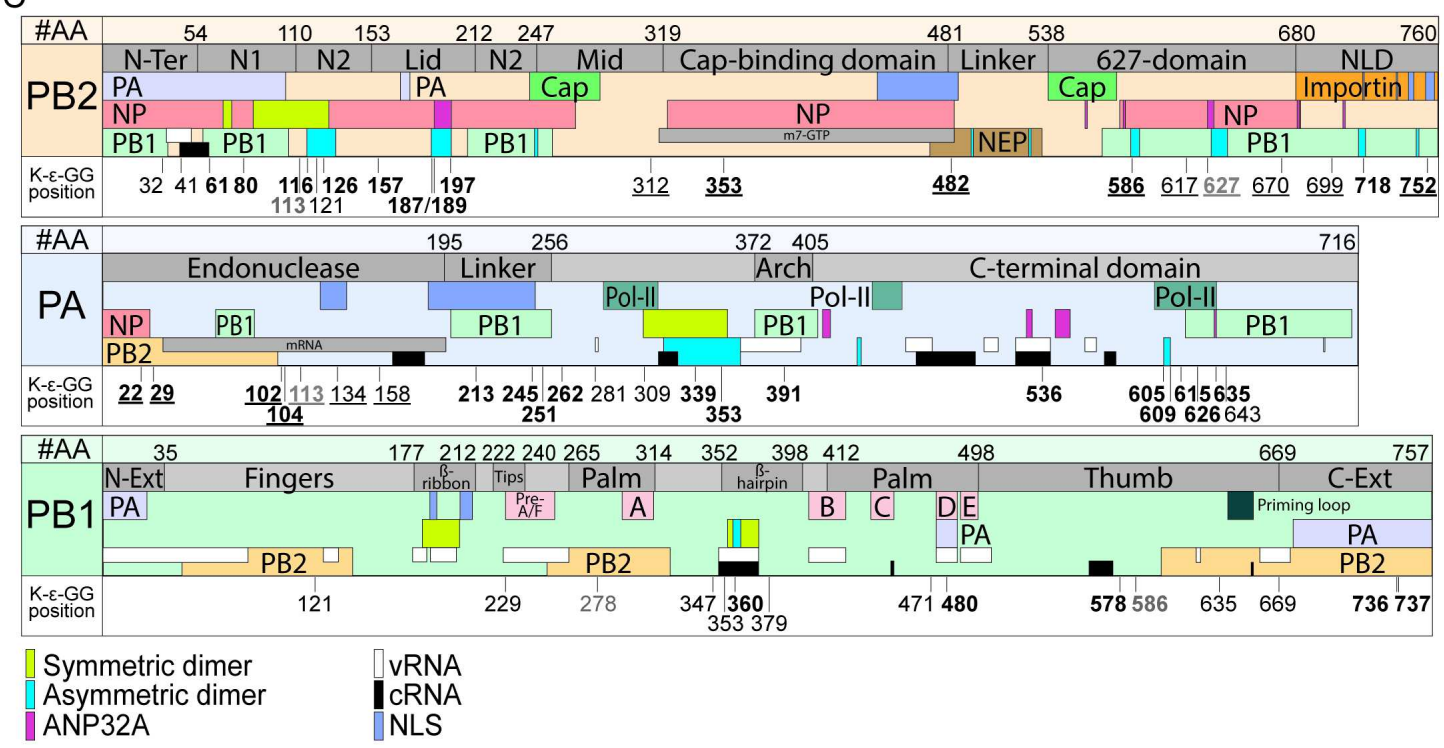

\section{Figure 2}

UB modified lysines reside in functional domains of the IAV polymerase 
Figure 3
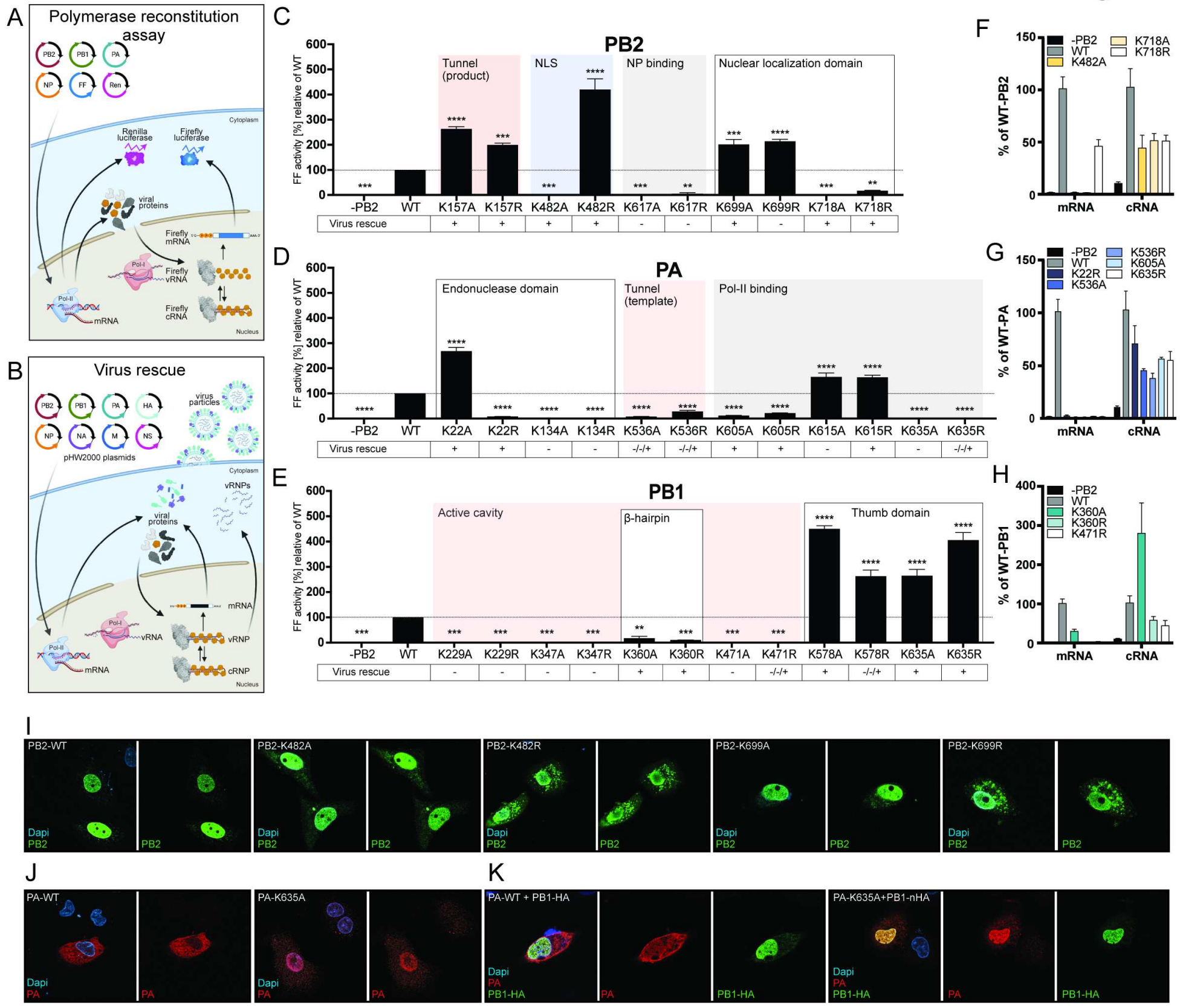

Figure 3

Mutational screen of modified lysines reveals distinct effects on mRNA transcription and vRNA replication 

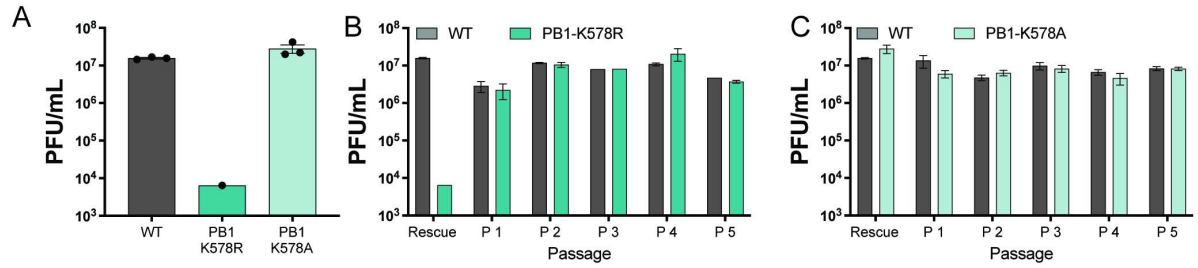

$\mathrm{D}$

\begin{tabular}{|c|c|c|c|c|c|c|c|c|c|c|c|c|c|c|c|c|c|}
\hline \multicolumn{3}{|c|}{ Nucleotide abundance } & \multicolumn{5}{|l|}{ R1 } & \multicolumn{5}{|l|}{ R2 } & \multicolumn{5}{|l|}{ R3 } \\
\hline & & & Position & A & $T$ & C & G & Position & A & $T$ & C & G & Position & A & $T$ & C & G \\
\hline \multirow[t]{3}{*}{$<5 \%$} & \multirow[t]{3}{*}{$5-50 \%$} & \multirow[t]{3}{*}{$50-95 \%>95$} & 1732 & 98 & 0 & 1 & 1 & 1732 & 0 & 0 & 71 & 29 & 1732 & 1 & 0 & 98 & 1 \\
\hline & & & 1733 & 99 & 0 & 1 & 0 & 1733 & 71 & 0 & 29 & 0 & 1733 & 97 & 0 & 1 & 2 \\
\hline & & & 1734 & 99 & 0 & 0 & 1 & 1734 & 30 & 0 & 0 & 70 & 1734 & 2 & 1 & 0 & 97 \\
\hline
\end{tabular}

E

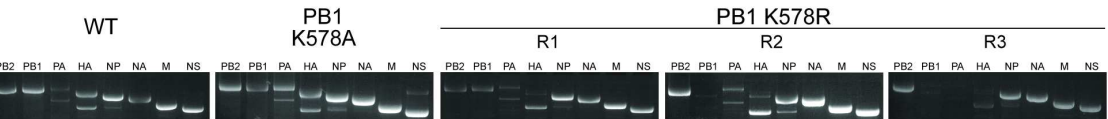

F

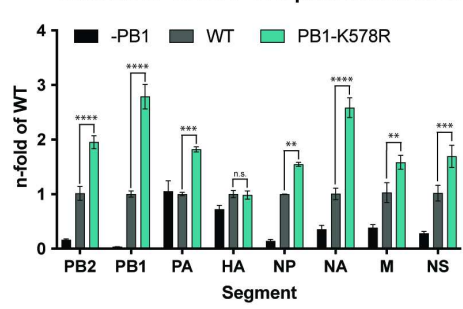

$\mathrm{G}$ VRNA in HEK293-T $48 \mathrm{~h}$ post transfection

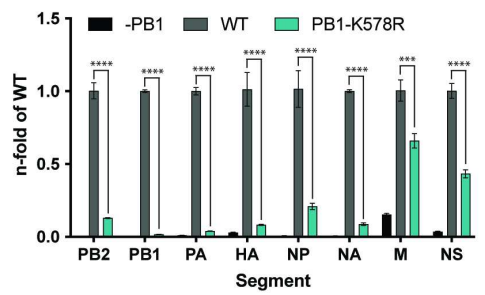

$\mathrm{H}$
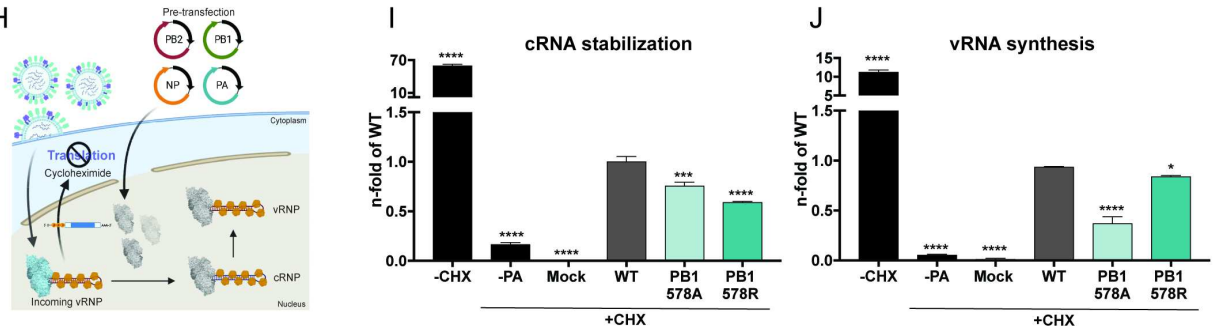

K

$\mathrm{L}$
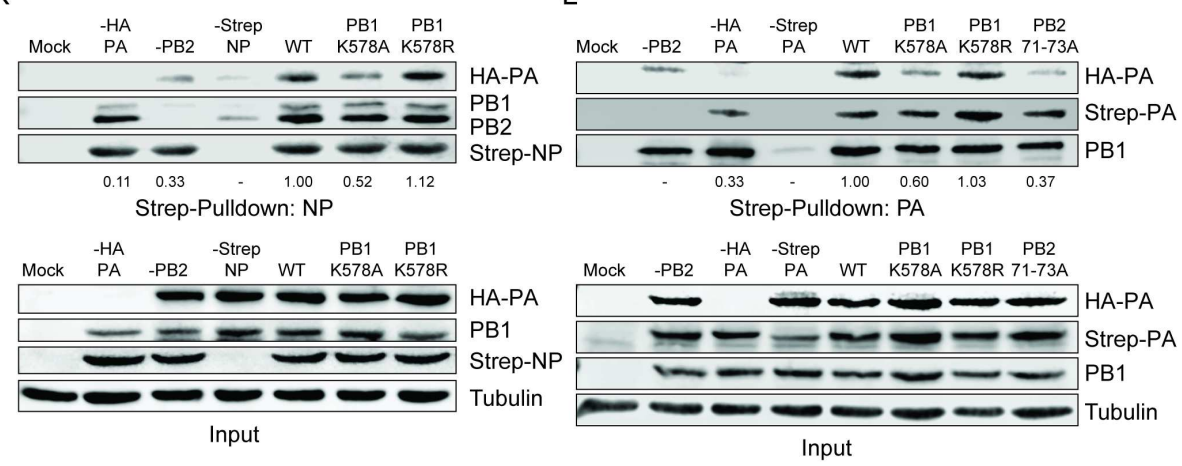

\section{Figure 4}

Mutation of PB1-K578 affects cRNP stabilization and vRNA transcription by modulating NP binding and polymerase dimerization 
Figure 5
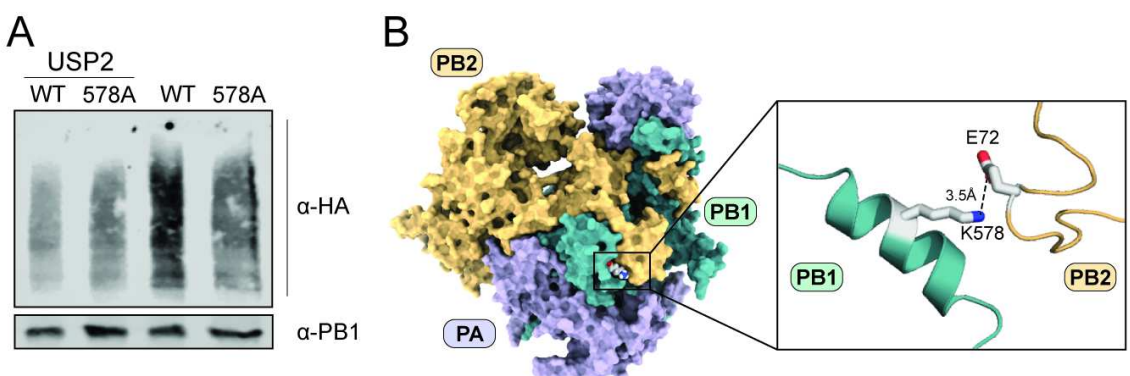

D Human

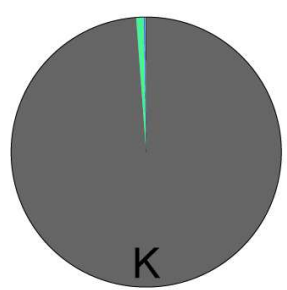

Avian

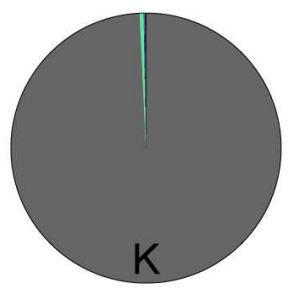

C

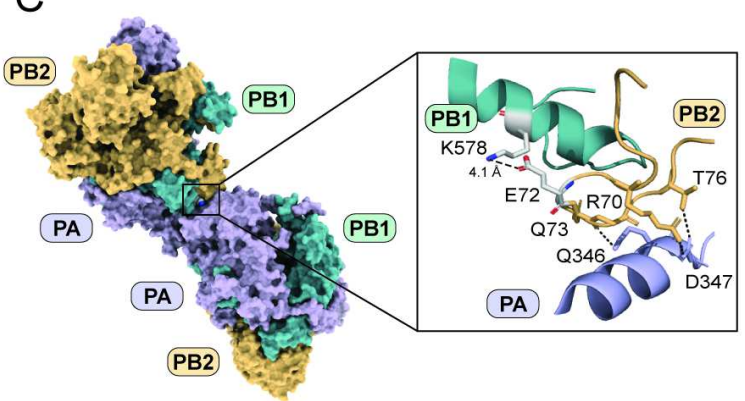

Swine

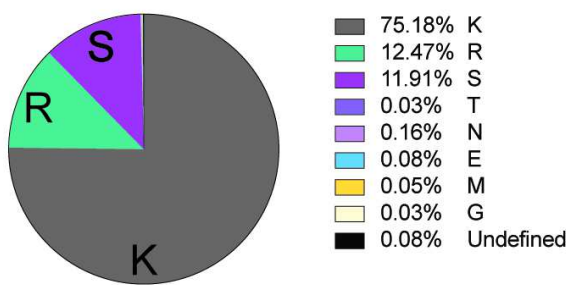

\section{E}

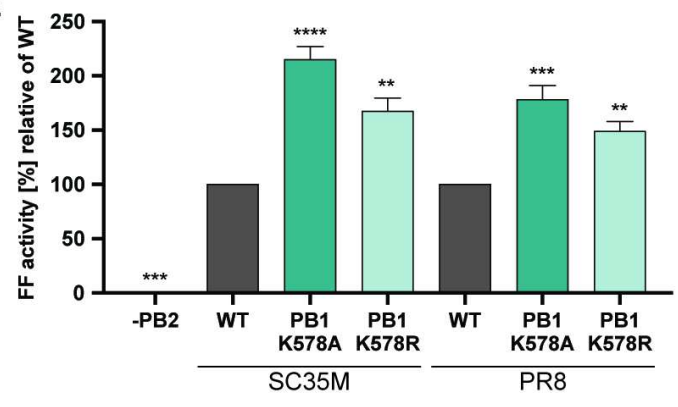

Figure 5

PB1-K578 is ubiquitinated and interacts with E72 in the PB2 N-terminus 
A

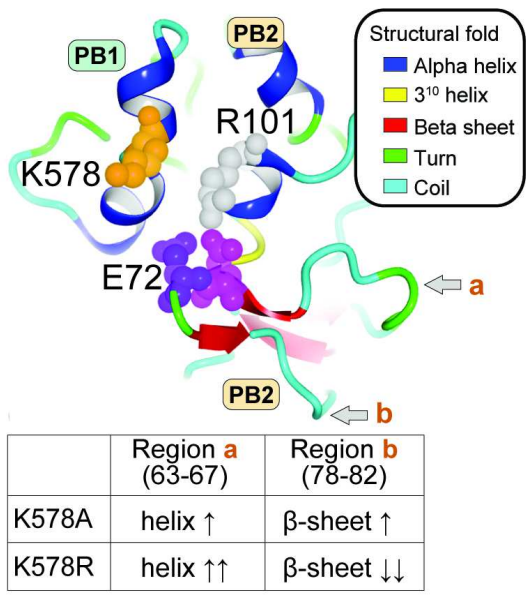

B

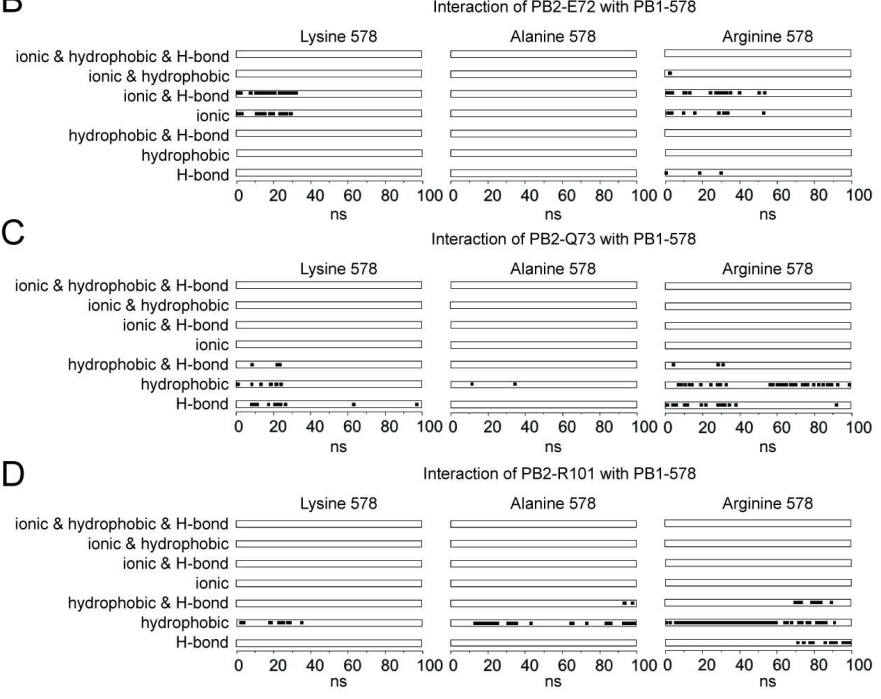

E

Primary mRNA transcription
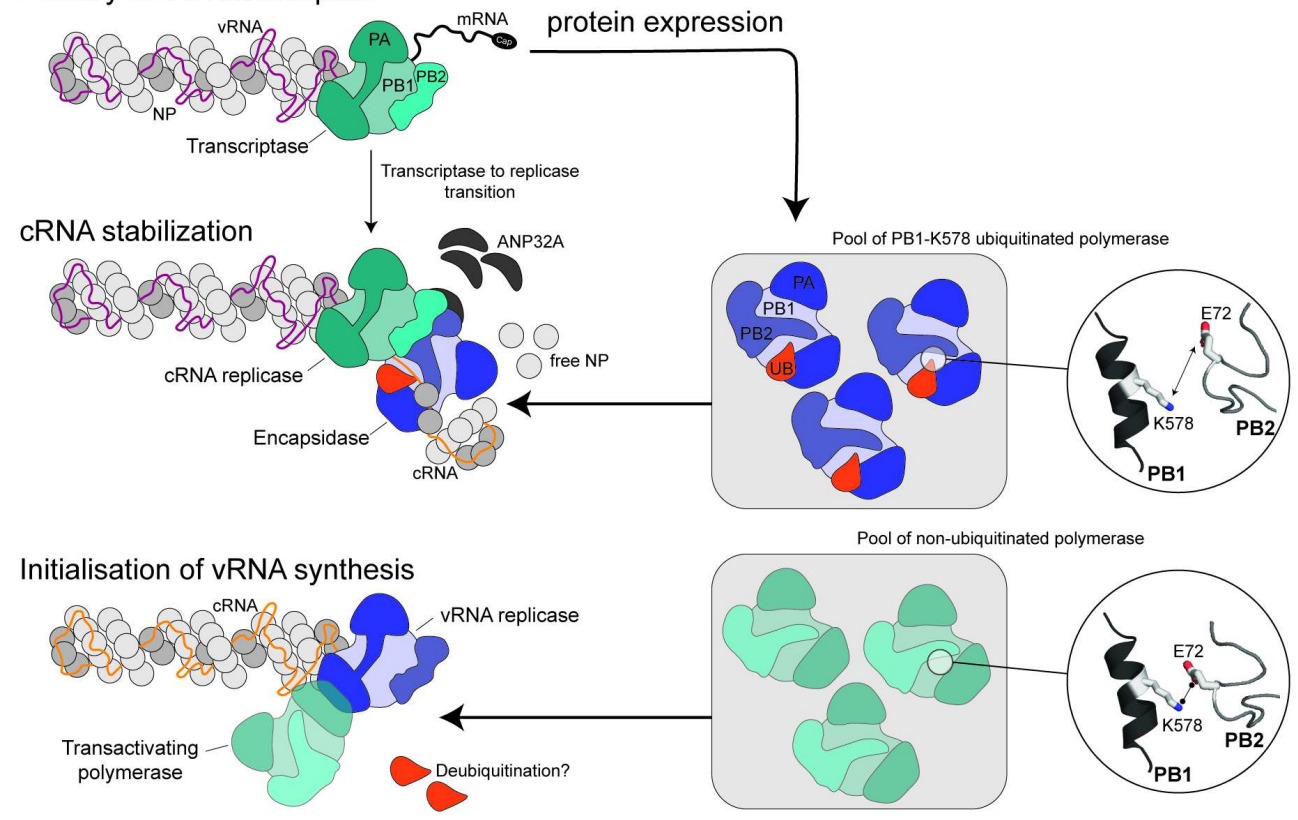

Pool of non-ubiquitinated polymerase

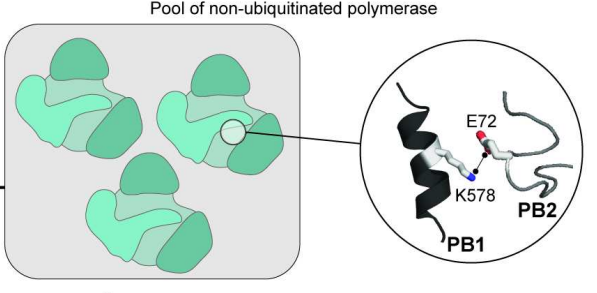

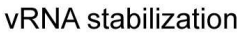

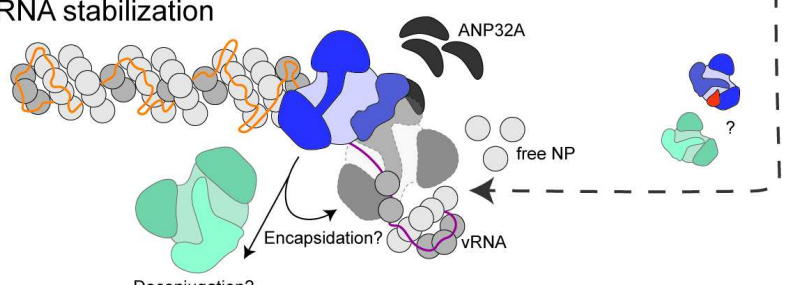

Deconjugation?

\section{Figure 6}

Interaction between PB1-K578 and PB2-E72 is dynamic and charge-dependent

\section{Supplementary Files}

This is a list of supplementary files associated with this preprint. Click to download. 
- NCOMMS2202428.pdf

- Supplementalinformation.pdf 